\title{
可见光催化炔硫醚的远程卤-二氟烷基化反应
}

\author{
朱海倩 ${ }^{a}$ 商甜波 ${ }^{a}$ 卢增辉 ${ }^{b}$ 罗 芳 ${ }^{a}$ 朱钢国 $*, a$ \\ ( ${ }^{a}$ 浙江师范大学化学系 先进催化材料教育部重点实验室 浙江金华 321004) \\ $\left({ }^{b}\right.$ 万州区生态环境监测站 重庆 404000)
}

\begin{abstract}
摘要 氟烷基取代的烯烃在生命科学及材料科学中具有重要价值. 炔烃的氟烷基化反应为氟烷基取代烯烃的合成提供 了有效方法，但是，目前大都属于 1,2-双官能团化反应，而炔烃的远程氟烷基化双官能团化反应依然有待发展. 以廉价 易得的卤代二氟烷基试剂为自由基前体，发展了一种可见光催化的炔硫醚远程卤一二氟烷基化反应，一步构建了远端 卤代的(Z)-氟烷基取代烯烃，其区域、立体和位点选择性优秀. 该反应条件温和，官能团兼容性良好，同时构建了 3 根 新的化学键, 为传统方法较难合成的、热力学相对不稳定的 $(Z)$-氟烷基取代烯烃提供了简单、高效的合成方法, 也为惰 性碳-氢键的直接卤代提供了新选择. 初步的机理研究表明, 反应经历了杂原子诱导的 $\beta$-氟烷基化、1,5-氢原子迁移、 单电子氧化和卤离子进攻的串联过程.
\end{abstract}

关键词 二氟烷基; 可见光催化; 炔硫醚; 碳-氢键卤代; 自由基; 选择性

\section{Visible-Light Photocatalytic Remote Halo-difluoroalkylation of Thioalkynes}

\author{
Zhu, Haiqian $^{a} \quad$ Shang, Tianbo $^{a} \quad$ Lu, Zenghui ${ }^{b} \quad$ Luo, Fang $^{a} \quad$ Zhu, Gangguo ${ }^{* a}$ \\ ( ${ }^{a}$ Key Laboratory of the Ministry of Education for Advanced Catalysis Materials, Department of Chemistry, \\ Zhejiang Normal University, Jinhua, Zhejiang 321004) \\ ( ${ }^{b}$ Wanzhou Ecological Environment Monitoring Station, Chongqing 404000)
}

\begin{abstract}
Fluoroalkylated alkenes are of significant importance in life sciences and functional materials. The fluoroalkylation of alkynes offers an efficient method for the synthesis of functionalized fluoroalkylated alkenes. However, the current methods are often limited to 1,2-difunctionalization, while the remote fluoroalkylative difunctionalization of alkynes has been rarely developed. Herein, a novel visible-light-induced remote halo-difluoroalkylation of thioalkynes is realized with difluoroalkyl halides as the radical source, forming distally halogenated ( $Z$ )-fluoroalkylated alkenes in moderate to high yields with excellent regio-, stereo-, and site-selectivity. The notable features of this reaction include the mild reaction conditions, broad substrate scope, concurrent formation of three new chemical bonds, and a thermodynamically less stable $(Z)$-alkene, thus enabling it a highly attractive method for organic synthesis. It represents a new advance on the direct $\mathrm{C}-\mathrm{H}$ bond halogenation. Preliminary mechanistic studies indicate a cascade radical process involving the heteroatom-induced $\beta$-fluoroalkylation of C-C triple bonds, intramolecular 1,5-hydrogen atom transfer (1,5-HAT), single electron transfer (SET) oxidation and halide addition.
\end{abstract}

Keywords difluoroalkyl; visible-light photoredox catalysis; thioalkyne; $\mathrm{C}-\mathrm{H}$ halogenation; radical; selectivity

由于氟原子独特的性质, 含氟有机化合物被广泛应 用于医药、农药、功能材料和生命科学等领域 ${ }^{[1]}$. 二氟 亚甲基的引入可以显著提高分子的代谢稳定性和口服 生物利用度, 已成为药物研发的一种重要手段 ${ }^{[2]}$. 近年 来, 炔烃的二氟烷基化反应引起了化学工作者的广泛关
注 $^{[3-12]}$. 炔烃的二氟烷基化双官能团化反应在引入二氟 烷基的同时，能够实现其它官能团的引入，具有良好的 步骤经济性和产物结构多样性, 可以快速构建官能团化 的氟烷基取代烯烃化合物库, 因而得到了快速发展 ${ }^{[4-12]}$. 例如, 胡金波等 ${ }^{[8]}$ 采用 $\mathrm{Et}_{3} \mathrm{~B}$ 为自由基引发剂, 实现了非

\footnotetext{
* Corresponding author. E-mail: gangguo@zjnu.cn

Received May 24, 2020; revised June 6, 2020; published online June 11, 2020

Dedicated to Professor Henry N. C. Wong on the occasion of his 70th birthday.

Project supported by the National Natural Science Foundation of China (No. 21672191), the Natural Science Foundation of Zhejiang Province (No. LZ20B020002) and the Education Department of Zhejiang Province (No. Y201942955).

国家自然科学基金(No. 21672191)、浙江省自然科学基金(No. LZ20B020002)和浙江省教育厅(No. Y201942955)资助项目.
} 
活化末端炔烃的碘-二氟烷基化反应(Scheme 1a). 采用

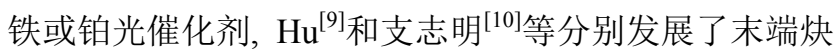
烃的反式一碘一二氟烷基化反应. 王细胜等 ${ }^{[1]}$ 通过铜催化 的自由基加成/脱羧串联反应, 实现了丙炔酸衍生物的 碘一二氟烷基化反应. 以 $\mathrm{CoBr}_{2}$ 为催化剂, von Wangelin 等 ${ }^{[12]}$ 发展了芳基炔烃的反式-溴-二氟烷基化反应, 进 一步拓展了卤一二氟烷基化的适用范围.

尽管炔烃的卤一烷基化反应取得了较快发展 ${ }^{[8-12]}$, 但仍然存在一些有待解决的问题. 首先, 传统方法通常 是末端炔烃的转化, 活性相对较低的非末端炔烃的反应 较为少见. 其次, 反应的选择性例如区域和立体选择性 有待提高. 更重要的是, 以往的反应属于 1,2 -双官能团 化, 而炔烃的远程卤一烷基化反应还未见报道. 最近, 我 们通过杂原子控制碳一碳参键氟烷基化的区域选择性, 相继实现了杂原子取代炔烃的远程氧化-氟烷基化 ${ }^{[13]}$ 和 炔基化一三氟甲基化反应 ${ }^{[14]}$, 为远端官能团化氟烷基取 代烯烃的合成提供了高效、高选择性的方法. 在以上工 作基础上, 本工作发展了一个可见光催化 ${ }^{[15]}$ 的炔硫醚 远程卤-二氟烷基化反应(Scheme 1b)，一步合成了远端 卤代的 $(Z)$-氟烷基取代烯烃, 反应的区域、立体和位点选 择性优秀, 为含氟功能分子的精准合成提供了有效途 径. 需要指出的是, 该反应实现了远程碳-氢键的卤 代 ${ }^{[16]}$, 包括澳代、碘代和氯代, 为惰性碳-氢键的直接卤 代提供了新思路. (a) previous reports on fluoroalkylative difunctionalization of alkynes

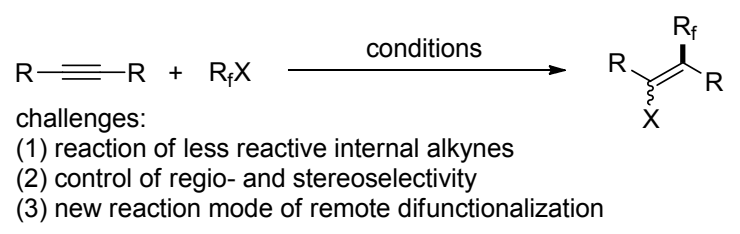

(b) this work: remote halo-fluoroalkylation of thioalkynes

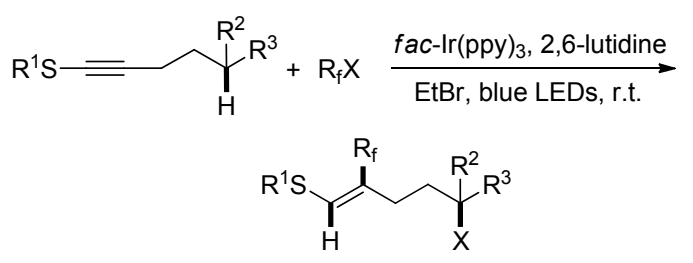

图式 1 炔烃的卤-氟烷基化反应

Scheme 1 Halo-fluoroalkylation of alkynes

\section{1 结果与讨论}

首先, 选取炔硫醚 ${ }^{[17]} \mathbf{1} \mathbf{a}$ 对反应条件进行了优化. 采 用 $\mathrm{BrCF}_{2} \mathrm{CO}_{2} \mathrm{Et}$ (2a, 2.0 equiv.) 为氟烷基自由基源, $\mathrm{fac}$ $\operatorname{Ir}(\mathrm{ppy})_{3}$ (2 mol\%)为光催化剂，2,6-二甲基吡啶 $(2.0$ equiv.)为添加剂, $15 \mathrm{~W}$ 蓝色 LED 灯为光源, 澳乙烷为溶 剂, 室温反应 $36 \mathrm{~h}$, 以 $72 \%$ 的产率得到远程溴一二氟烷 基产物 3aa(表 1, Entry 1). 采用 $\mathrm{Ru}(\mathrm{bpy})_{3} \mathrm{Cl}_{2}$ 为光催化剂 则导致反应效率和选择性明显下降(Entry 2). 接下来, 以 $f a c-\operatorname{Ir}(\mathrm{ppy})_{3}$ 为光催化剂, 对反应添加剂进行了优化. 结果发现，吡啶、2,4,6-三甲基吡啶、醋酸钾、碳酸钾和

表 1 炔硫醚远程溴-二氟烷基化反应条件优化 ${ }^{a}$

Table 1 Optimization of reaction conditions for the remote hlao-difluoroalkylation of thioalkynes

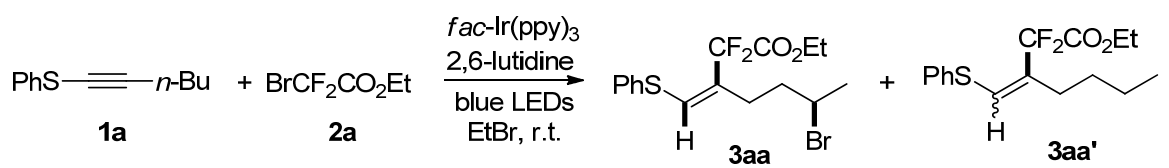

\begin{tabular}{|c|c|c|}
\hline Entry & Deviations from standard condition & Yield $^{b} / \%$ of $\mathbf{3 a a} / \mathbf{3 a a}$ \\
\hline 1 & None & $72 /<5$ \\
\hline 2 & $\mathrm{Ru}(\mathrm{bpy})_{3} \mathrm{Cl}_{2}$ instead of $f a c-\operatorname{Ir}(\mathrm{ppy})_{3}$ & $25 / 5$ \\
\hline 3 & $\mathrm{Et}_{3} \mathrm{~N}$ instead of 2,6-lutidine & $65 / 9$ \\
\hline 4 & Pyridine instead of 2,6-lutidine & $61 / 7$ \\
\hline 5 & $2,4,6$-collidine instead of 2,6-lutidine & $55 / 4$ \\
\hline 6 & KOAc instead of 2,6-lutidine & $70 / 8$ \\
\hline 7 & $\mathrm{~K}_{2} \mathrm{CO}_{3}$ instead of 2,6-lutidine & $62 / 4$ \\
\hline 8 & $\mathrm{~K}_{2} \mathrm{HPO}_{4}$ instead of 2,6-lutidine & $68 / 6$ \\
\hline 9 & $\mathrm{MeCN}$ instead of $\mathrm{EtBr}$ & $69 / 10$ \\
\hline 10 & DMSO instead of EtBr & $70 / 7$ \\
\hline 11 & DCM instead of EtBr & $69 / 15$ \\
\hline 12 & THF instead of EtBr & $7 / 2$ \\
\hline 13 & Without 2,6-lutidine & $9 / 4$ \\
\hline 14 & Without $f a c-\operatorname{Ir}(\mathrm{ppy})_{3}$ & NR \\
\hline
\end{tabular}

${ }^{a}$ Reaction conditions: 1a $(0.2 \mathrm{mmol}), \mathbf{2 a}(0.4 \mathrm{mmol}), f a c-\mathrm{Ir}(\mathrm{ppy}){ }_{3}(2 \mathrm{~mol} \%), 2,6$-lutidine $(0.4 \mathrm{mmol}), \mathrm{EtBr}(2 \mathrm{~mL}), 25{ }^{\circ} \mathrm{C}, 15 \mathrm{~W}$ blue LEDs, $36 \mathrm{~h} .{ }^{b}$ Isolated yield. $\mathrm{NR}=$ No reaction. 
磷酸氢二钾等都不如 2,6-二甲基吡啶(Entries 3～8). 采 用 $\mathrm{MeCN}$ 、二甲基亚砜(DMSO)或二氯甲烷(DCM)为溶 剂, 3aa 的产率变化不大, 但是, 氢二氟烷基化副产物 3aa'开始增多(Entries 9１1). 控制实验表明, 无论 2,6二甲基吡啶还是 $f a c-\operatorname{Ir}(\mathrm{ppy})_{3}$, 对该反应都起着重要作用 (Entries 13, 14).

在实现优化反应条件之后, 对杂原子取代炔烃的底 物适用范围进行了研究(表 2). 首先, 考察了硫取代基对 反应的影响. 无论芳基炔硫醚还是脂肪基炔硫醚，反应 都能很好地进行, 以良好的产率得到远程溴-二氟烷基 化产物 $(3 \mathrm{aa} \sim 3 \mathrm{ea})$, 反应的区域、立体和位点选择性优 秀. 延长远端碳链的长度, 依然可以得到很好的收率 (3fa，3ga). 接下来，考察了反应的官能团兼容性. 结果 发现, 醚(OTBS, OMe)、酯基 $(\mathrm{OAc})$ 、卤原子 $(\mathrm{Br}, \mathrm{Cl})$ 和酰 胺 $(\mathrm{NPhth})$ 都能很好地兼容 $(\mathbf{3 h a} \sim \mathbf{3 m a})$, 这些基团为产 物的进一步衍生化提供了可能. 炔丙位及高炔丙位取代 基的引入不会影响反应的进行(3na, 3oa). 除了二级碳氢键直接溴化之外, 三级碳一氢键也能顺利转化, 得到
相应的溴代产物 $3 \mathrm{pa}$, 不过反应伴随着少量氢二氟烷基 化产物 $3 \mathrm{pa}^{\prime}$ 和消除产物 $3 \mathbf{p a}^{\prime \prime}$ 的生成. 此外, 茮位碳一氢 键的溴代反应也能顺利进行(3ra). 该远程溴-二氟烷基 化反应可以拓展到炔基硒化合物，而炔醚在该反应条件 下则基本不反应(3ta, 3ua).

接下来，以 $1 \mathrm{a}$ 为偶联试剂，对自由基前体进行了研 究(表 3 ). 令人高兴的是, 各种溴代二氟乙酰胺都能顺利 反应，以中等到良好的产率得到远程溴-二氟烷基化产 物(3ab 3af), 反应的区域、立体和位点选择性优秀. 以 Umemoto 试剂为三氟甲基源, $\mathrm{NaBr}$ 或 $\mathrm{NaI}$ 为卤离子源, 分别实现了炔硫醚的远程溴-三氟甲基或碘-三氟甲基 化反应(3ah, 3ai)(产率未进一步优化), 该结果说明反应 可能经历碳正离子中间体. 令人高兴的是，其它自由基 前体也能参与该反应. 例如，对甲基苯磺酰氯( $\mathrm{TsCl}$ )为 偶联试剂，以 $57 \%$ 的产率得到了远程氯一磺化产物(3aj).

然后，对该反应机理进行了研究. 在标准反应条件 下，加入 2.0 equiv.的自由基捕获试剂四甲基哌啶氧化物 (TEMPO), 反应被完全抑制, 并观察到了 $\mathbf{4 a}^{[18]}$ 的生成

表 2 杂原子取代炔烃的底物范围 ${ }^{a}$

Table 2 Substrate scope of heteroalkynes

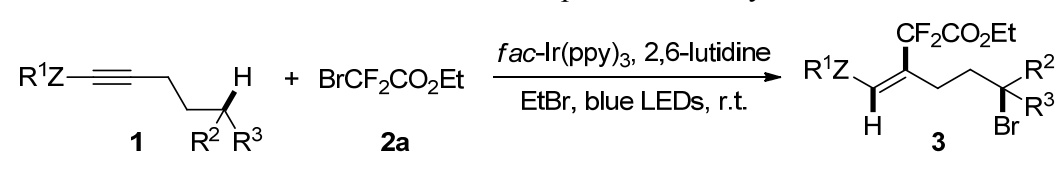

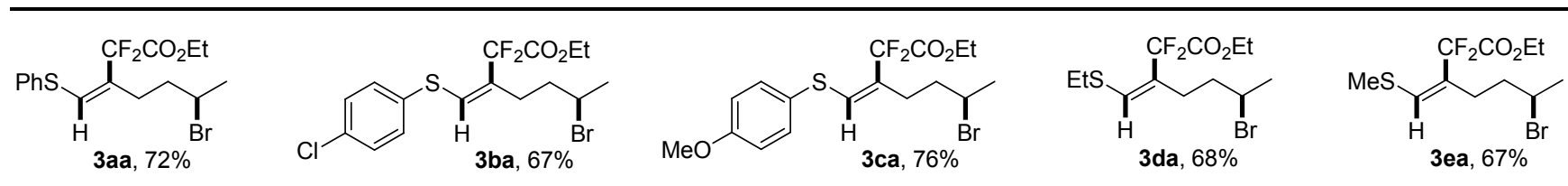<smiles>CCOC(=O)C(=Cc1ccccc1)C(F)(F)F</smiles>
$3 \mathrm{fa}, 80 \%$<smiles>CCCC(CCC(Br)C=Cc1ccccc1)(C(=O)OCC)C(F)(F)F</smiles>
3ga, $70 \%$<smiles>CCOC(=O)C(=Cc1ccccc1)CCC(Br)CCBr</smiles>

3ja, $64 \%$<smiles>CCOC(=O)C(=Cc1ccccc1)CCC(Br)CCCl</smiles>

3ka, $55 \%$<smiles>CCOC(=O)OCCC(Br)C(=Cc1ccccc1)C(F)(F)F</smiles>

3ha, $56 \%$<smiles>CCOC(=O)C(=Cc1ccccc1)CCC(Br)CC[NH+]c1ccccc1</smiles>
3la, $51 \%$<smiles>CCOC(=O)C(=CC(=O)c1ccccc1)CCC(Br)CCOC(C)=O</smiles>

3ia, $48 \%$<smiles>C/C=C(\CC1CCCCC1Br)C(F)(F)C(=O)OCC</smiles><smiles>CC=C(C)C(O[18O])C(CC(Br)CC)C(=O)OCC</smiles><smiles>CCOC(=O)C(F)(F)/C(=C\C(=P)c1ccccc1)CC(C)(F)CBr</smiles>

3qa, $37 \%$<smiles>CCOC(=O)C(=CC(C)(C)C)CCC(Br)c1ccccc1</smiles>

3ra, $62 \%^{e}$<smiles>CCOC(=O)C(C)=C(CCC(C)(Br)CC)C(C)(Br)Br</smiles>

3pa, $55 \%$<smiles>CCOC(=O)C(CCC(C)C)=C(C)C</smiles>

3pa', 10\%<smiles>CCOC(=O)C(CCC(Br)CCOC)=C(C)C</smiles>

3ma, $62 \%$

\footnotetext{
${ }^{a}$ Reaction conditions: $1(0.2 \mathrm{mmol}), 2 \mathrm{a}(0.4 \mathrm{mmol}), f a c-\mathrm{Ir}(\mathrm{ppy})_{3}(2 \mathrm{~mol} \%), 2,6$-lutidine $(0.4 \mathrm{mmol}), \mathrm{EtBr}$, blue LEDs, $25{ }^{\circ} \mathrm{C}, 36 \mathrm{~h}$; isolated yields are given. ${ }^{b} d r=$ $1.8: 1 .{ }^{c} d r=1.5: 1 .{ }^{d}$ Determined by GC-MS. ${ }^{e}$ Similar with the reaction of $1 \mathbf{p}, 11 \%$ yield of hydrodifluoroalkylation product 3 ra' and $<5 \%$ yield of remote desaturated product 3ra" was observed by GC-MS. ${ }^{f} 14 \%$ yield of hydrodifluoroalkylation product 3sa' was observed by GC-MS.
} 
表 3 自由基前体范围 ${ }^{a}$

Table 3 Scope of radical precursors

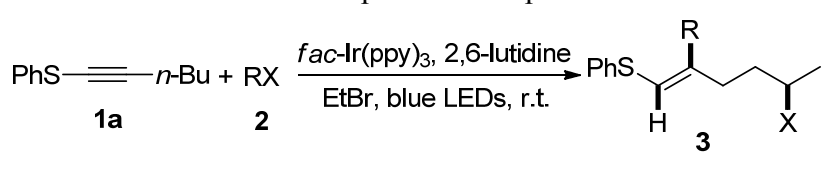<smiles>CC(Br)CC/C(=C/Sc1ccccc1)C(F)(F)C(=O)N1CC[N]CC1</smiles>

3ab, $71 \%$<smiles>CCN(CC)C(=O)C(F)(F)/C(=C\C(=S)c1ccccc1)CCC(C)Br</smiles>

3ae, $43 \%$<smiles>CC(Br)CC/C(=C/c1ccccc1)C(F)(F)F</smiles>

3ah, $42 \%^{b}$<smiles></smiles>

3ac, $74 \%$<smiles>CC(Br)CC/C(=C/c1ccccc1)C(F)(F)C(N)=O</smiles>

3af, $54 \%$<smiles>CC(I)CC/C(=C/c1ccccc1)C(F)(F)F</smiles>

3ai, $30 \%^{b}$<smiles>CC(Br)CC/C=C(/C=Pc1ccccc1)C(F)(F)C(=O)N1CCOCC1</smiles>

3ad, $61 \%$<smiles>CCCCC/C=C(/CCC(C)Br)C(F)(F)C(=O)OC</smiles>

3ag, $75 \%$<smiles>CC(Cl)CC/C([13I])=C\c1ccccc1</smiles>

3aj, $57 \%$

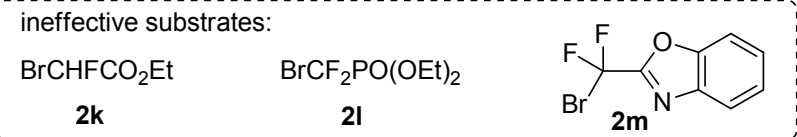

$\overline{{ }^{a}}$ Reaction conditions: 1a $(0.2 \mathrm{mmol}), 2(0.4 \mathrm{mmol}), f a c-\operatorname{Ir}(\mathrm{ppy})_{3}(2 \mathrm{~mol} \%)$, 2,6-lutidine $(0.4 \mathrm{mmol}), \mathrm{EtBr}$, blue LEDs, $25{ }^{\circ} \mathrm{C}, 36 \mathrm{~h}$; isolated yields are given. ${ }^{b}$ Umemoto's reagent $(0.4 \mathrm{mmol}), \mathrm{NaX}(0.6 \mathrm{mmol})$, and $\mathrm{ClCH}_{2} \mathrm{CH}_{2} \mathrm{Cl}$ were used instead of $\mathbf{2 a}$ and $\mathrm{EtBr}$.

$$
\begin{aligned}
& \mathrm{PhS}=n-\mathrm{Bu}+\mathrm{BrCF}_{2} \mathrm{CO}_{2} \mathrm{Et} \stackrel{\begin{array}{l}
\text { TEMPO (2.0 equiv.) } \\
\text { standard conditions }
\end{array}}{\longrightarrow} \\
& \text { 1a 2a } \\
& \text { 3aa, } 0 \% 0 \\
& \mathrm{PhS}=n-\mathrm{Bu}+\mathrm{BrCF}_{2} \mathrm{CO}_{2} \mathrm{Et} \stackrel{\begin{array}{c}
\mathrm{MeOH}(2.0 \text { equiv. }) \\
\text { standard conditions }
\end{array}}{\longrightarrow} \\
& \text { 1a } \\
& \text { 2a } \\
& \text { 3aa, } 65 \% \text { Br }
\end{aligned}
$$

图式 2 机理研究

Scheme 2 Mechanistic studies

(Scheme 2), 说明反应可能经历二氟烷基自由基中间体. 在反应体系加入 2.0 equiv. $\mathrm{MeOH}$, 高分辨质谱检测到 了远程碳-氢键甲氧基化产物 $4 \mathrm{~b}$, 再次说明反应可能经
历碳正离子中间体. 开关灯实验表明(图 1), 光照对反应 的进行是必须的.

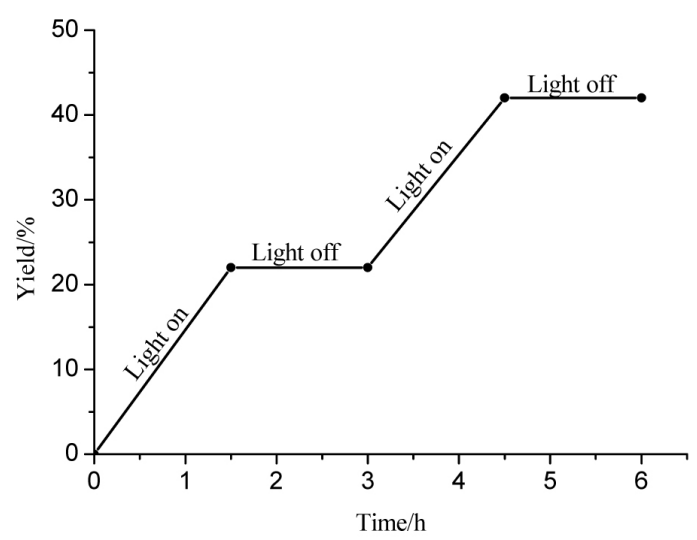

图 1 化合物 $1 \mathrm{a}$ 和 $2 \mathrm{a}$ 反应的开关灯实验

Figure 1 Light on/off experiment of the reaction between 1a and 2a

根据上述实验结果和我们之前的工作 ${ }^{[13-14]}$, 可能的 反应机理如 Scheme 3 所示. 首先, $f a c-\operatorname{Ir}(\mathrm{ppy})_{3}$ 经可见光 照射转化为激发态的* $\operatorname{Ir}(\mathrm{III})$, 接着, 它与 $\mathrm{R}_{\mathrm{f}} \mathrm{X}$ 单电子转 移(SET)产生氟烷基自由基 $\mathrm{R}_{\mathrm{f}}{ }^{\circ[19]}$. 硫原子对碳一碳参键 的给电子共轭效应将使得电子密度较高的 $\beta$-炔碳原子 选择性与亲电氟烷基自由基结合，生成烯基自由基 $\mathbf{I}$; 当然，硫原子的孤对电子对邻位烯基自由基的共轭作用 也会有利于 $\mathbf{I}$ 的生成. 随后，烯基自由基分子内 1,5 -氢

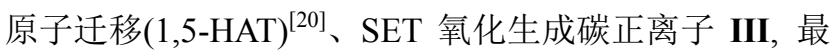
后，卤离子进攻碳正离子得到相应的远程卤一氟烷基化 产物, 同时再生光催化剂 $\operatorname{Ir}(\mathrm{III})$.

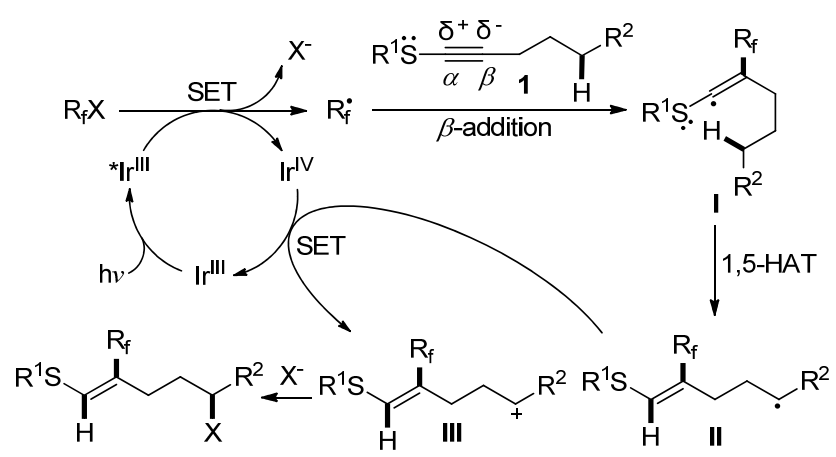

图式 3 可能的反应机理

Scheme 3 Possible reaction mechanism

\section{2 结论}

发展了一个可见光催化的炔硫醚远程卤-二氟烷基 化反应，一步合成了远端卤代的(Z)-二氟烷基取代的烯 基硫醚，产率中等到良好，反应的区域、立体和位点选 择性优秀. 反应一步构建了 3 根新的化学键, 实现了惰 性碳-氢键的直接卤代，包括溴代、碘代和氯代，同时构 
建了热力学相对不稳定的 ( $Z$ )-烯烃, 为复杂分子的精准 合成提供了温和高效的方法.

\section{3 实验部分}

\section{1 仪器与试剂}

${ }^{1} \mathrm{H}$ NMR、 ${ }^{13} \mathrm{C}$ NMR 和 ${ }^{19} \mathrm{~F}$ NMR 谱采用德国布鲁克 公司 600 或 $400 \mathrm{MHz}$ 核磁共振仪进行测定. 高分辨质谱 采用布鲁克公司 MicroTof-Q II 液质联用高分辨质谱仪 测定，离子源为 ESI-TOF. 用硅胶 (200 300 目)进行柱 层析分离. 除非另有说明, 所有试剂和溶剂均购自商业 供应商, 未经进一步纯化.

\section{2 实验方法}

在干燥的 $15 \mathrm{~mL}$ 反应管中, 氮气保护下加入 $f a c-$ $\operatorname{Ir}(\text { ppy })_{3}(2.6 \mathrm{mg}, 0.004 \mathrm{mmol}), 1(0.2 \mathrm{mmol}), 2$ (0.4 $\mathrm{mmol}$ ), 2,6-二甲基吡啶( $42.9 \mathrm{mg}, 0.4 \mathrm{mmol}$ )和 $2 \mathrm{~mL}$ 干燥 的澳乙烷, 距离反应管约 $5 \mathrm{~cm}$ 处用 $15 \mathrm{~W}$ 蓝色 LED 灯 $25{ }^{\circ} \mathrm{C}$ 下照射摚拌 $36 \mathrm{~h}$. 待反应结束后, 加入 $5 \mathrm{~mL}$ 水淬 灭反应, 再用乙酸乙酯 $(10 \mathrm{~mL} \times 4)$ 萃取, 有机相用饱和 氯化钠溶液洗涤, 无水硫酸钠干燥, 旋转蒸发除去溶剂. 粗产品用 $200 \sim 300$ 目硅胶柱层析 $V($ ( 酸乙酯)：V(石油 醚 $)=1 ： 30$ ]分离, 得到目标产物 3 .

3-苯硫基亚甲基-2,2-二氟-6-澳庚酸乙酯 (3aa): 56 $\mathrm{mg}$, 黄色液体, 产率 $72 \% .{ }^{1} \mathrm{H} \mathrm{NMR}\left(600 \mathrm{MHz}, \mathrm{CDCl}_{3}\right) \delta$ : $7.35 \sim 7.33(\mathrm{~m}, 4 \mathrm{H}), 7.31 \sim 7.28(\mathrm{~m}, 1 \mathrm{H}), 6.61(\mathrm{~s}, 1 \mathrm{H})$, $4.33(\mathrm{q}, J=7.1 \mathrm{~Hz}, 2 \mathrm{H}), 4.20 \sim 4.09(\mathrm{~m}, 1 \mathrm{H}), 2.64 \sim 2.56$ $(\mathrm{m}, 1 \mathrm{H}), 2.51 \sim 2.39(\mathrm{~m}, 1 \mathrm{H}), 2.05 \sim 1.92(\mathrm{~m}, 2 \mathrm{H}), 1.75(\mathrm{~d}$, $J=6.7 \mathrm{~Hz}, 3 \mathrm{H}), 1.33(\mathrm{t}, J=7.1 \mathrm{~Hz}, 3 \mathrm{H}) ;{ }^{13} \mathrm{C}$ NMR $(151$ $\left.\mathrm{MHz}, \mathrm{CDCl}_{3}\right) \delta: 163.4(\mathrm{t}, J=34.6 \mathrm{~Hz}), 135.0,131.3(\mathrm{t}, J=$ $5.8 \mathrm{~Hz}), 130.7(\mathrm{t}, J=23.5 \mathrm{~Hz}), 123.0,129.3,127.6,113.6$ (t, $J=252.1 \mathrm{~Hz}), 63.2,50.5,39.6,31.4(\mathrm{t}, J=3.3 \mathrm{~Hz})$, $26.5,13.9 ;{ }^{19} \mathrm{~F}$ NMR $\left(565 \mathrm{MHz}, \mathrm{CDCl}_{3}\right) \delta:-99.0(\mathrm{~d}, J=$ $273.1 \mathrm{~Hz}),-99.6(\mathrm{~d}, J=273.1 \mathrm{~Hz})$; HRMS (ESI) calcd for $\mathrm{C}_{16} \mathrm{H}_{20} \mathrm{BrF}_{2} \mathrm{O}_{2} \mathrm{~S}(\mathrm{M}+\mathrm{H})^{+}$393.0330, found 393.0323.

3-对氯苯硫基亚甲基-2,2-二氟-6-溴庚酸乙酯 (3ba): $57 \mathrm{mg}$, 黄色液体, 产率 67\%. ${ }^{1} \mathrm{H}$ NMR (600 MHz, $\left.\mathrm{CDCl}_{3}\right) \delta: 7.33 \sim 7.31(\mathrm{~m}, 2 \mathrm{H}), 7.29 \sim 7.27(\mathrm{~m}, 2 \mathrm{H}), 6.54$ (s, 1H), $4.33(\mathrm{q}, J=7.2 \mathrm{~Hz}, 2 \mathrm{H}), 4.17 \sim 4.12(\mathrm{~m}, 1 \mathrm{H})$, $2.63 \sim 2.59(\mathrm{~m}, 1 \mathrm{H}), 2.49 \sim 2.44(\mathrm{~m}, 1 \mathrm{H}), 2.03 \sim 1.91(\mathrm{~m}$, $2 \mathrm{H}), 1.75(\mathrm{~d}, J=6.7 \mathrm{~Hz}, 3 \mathrm{H}), 1.33(\mathrm{t}, J=7.1 \mathrm{~Hz}, 3 \mathrm{H}) ;{ }^{13} \mathrm{C}$ NMR (151 MHz, $\left.\mathrm{CDCl}_{3}\right) \delta: 163.3$ (t, $\left.J=34.5 \mathrm{~Hz}\right), 133.8$, 133.4, 131.5 (t, $J=23.6 \mathrm{~Hz}), 131.2,130.5(\mathrm{t}, J=5.7 \mathrm{~Hz})$, $129.4,113.5(\mathrm{t}, J=252.3 \mathrm{~Hz}), 63.3,50.4,39.5,31.4(\mathrm{t}, J=$ $3.5 \mathrm{~Hz}), 26.5,13.9 ;{ }^{19} \mathrm{~F}$ NMR $\left(565 \mathrm{MHz}, \mathrm{CDCl}_{3}\right) \delta$ : -99.0 (d, $J=273.5 \mathrm{~Hz}),-99.7$ (d, $J=273.4 \mathrm{~Hz})$; HRMS (ESI) calcd for $\mathrm{C}_{16} \mathrm{H}_{18} \mathrm{BrClF}_{2} \mathrm{O}_{2} \mathrm{SNa}(\mathrm{M}+\mathrm{Na})^{+} 448.9760$, found 448.9758 .

3-对甲氧基苯硫基亚甲基-2,2-二氟-6-溴庚酸乙酯 (3ca): $64 \mathrm{mg}$, 黄色液体, 产率 76\%. ${ }^{1} \mathrm{H} \mathrm{NMR}$ (400 MHz, $\left.\mathrm{CDCl}_{3}\right) \delta: 7.32 \sim 7.30(\mathrm{~m}, 2 \mathrm{H}), 6.89 \sim 6.86(\mathrm{~m}, 2 \mathrm{H}), 6.51$ $(\mathrm{s}, 1 \mathrm{H}), 4.36(\mathrm{q}, J=7.1 \mathrm{~Hz}, 2 \mathrm{H}), 4.17 \sim 4.09(\mathrm{~m}, 1 \mathrm{H}), 3.81$ $(\mathrm{s}, 3 \mathrm{H}), 2.59 \sim 2.52(\mathrm{~m}, 1 \mathrm{H}), 2.44 \sim 2.37(\mathrm{~m}, 1 \mathrm{H}), 2.03 \sim$ $1.88(\mathrm{~m}, 2 \mathrm{H}), 1.73(\mathrm{~d}, J=6.7 \mathrm{~Hz}, 3 \mathrm{H}), 1.36(\mathrm{t}, J=7.1 \mathrm{~Hz}$, $3 \mathrm{H}) ;{ }^{13} \mathrm{C}$ NMR $\left(151 \mathrm{MHz}, \mathrm{CDCl}_{3}\right) \delta: 163.5(\mathrm{t}, J=34.7$ $\mathrm{Hz}), 159.7,133.4(\mathrm{t}, J=5.8 \mathrm{~Hz}), 132.8,128.7(\mathrm{t}, J=23.6$ $\mathrm{Hz}), 125.4,114.9,113.7$ (t, $J=252.0 \mathrm{~Hz}), 63.1,55.4,50.5$, $39.7,31.2(\mathrm{t}, J=3.5 \mathrm{~Hz}), 26.4,13.9 ;{ }^{19} \mathrm{~F}$ NMR $(565 \mathrm{MHz}$, $\left.\mathrm{CDCl}_{3}\right) \delta:-99.2(\mathrm{~d}, J=271.8 \mathrm{~Hz}),-99.8(\mathrm{~d}, J=271.8$ $\mathrm{Hz}$ ); HRMS (ESI) calcd for $\mathrm{C}_{17} \mathrm{H}_{21} \mathrm{BrF}_{2} \mathrm{O}_{3} \mathrm{SNa}(\mathrm{M}+\mathrm{Na})^{+}$ 445.0255, found 445.0262 .

3-乙硫基亚甲基-2,2-二氟-6-溴庚酸乙酯(3da)：47 $\mathrm{mg}$, 黄色液体, 产率 $68 \% .{ }^{1} \mathrm{H} \mathrm{NMR}\left(400 \mathrm{MHz}, \mathrm{CDCl}_{3}\right) \delta$ : 6.40 (s, 1H), 4.34 (q, $J=7.1 \mathrm{~Hz}, 2 \mathrm{H}), 4.17 \sim 4.09(\mathrm{~m}, 1 \mathrm{H})$, $2.71(\mathrm{q}, J=7.4 \mathrm{~Hz}, 2 \mathrm{H}), 2.57 \sim 2.51(\mathrm{~m}, 1 \mathrm{H}), 2.44 \sim 2.36$ $(\mathrm{m}, 1 \mathrm{H}), 2.02 \sim 1.86(\mathrm{~m}, 2 \mathrm{H}), 1.74(\mathrm{~d}, J=6.7 \mathrm{~Hz}, 3 \mathrm{H})$, $1.36(\mathrm{t}, J=7.1 \mathrm{~Hz}, 3 \mathrm{H}), 1.30(\mathrm{t}, J=7.4 \mathrm{~Hz}, 3 \mathrm{H}) ;{ }^{13} \mathrm{C} \mathrm{NMR}$ $\left(151 \mathrm{MHz}, \mathrm{CDCl}_{3}\right) \delta: 163.5(\mathrm{t}, J=34.9 \mathrm{~Hz}), 132.9(\mathrm{t}, J=$ $6.1 \mathrm{~Hz}), 127.9(\mathrm{t}, J=23.5 \mathrm{~Hz}), 113.7$ (t, $J=251.6 \mathrm{~Hz})$, 63.0, 50.7, 39.8, 31.4 (t, $J=3.2 \mathrm{~Hz}), 29.2,26.5,15.3,13.9$; ${ }^{19} \mathrm{~F} \mathrm{NMR}\left(565 \mathrm{MHz}, \mathrm{CDCl}_{3}\right) \delta:-100.2(\mathrm{~d}, J=269.7 \mathrm{~Hz})$, $-100.8\left(\mathrm{~d}, J=269.8 \mathrm{~Hz}\right.$ ); HRMS (ESI) calcd for $\mathrm{C}_{12} \mathrm{H}_{19^{-}}$ $\mathrm{BrF}_{2} \mathrm{O}_{2} \mathrm{SNa}(\mathrm{M}+\mathrm{Na})^{+}$367.0149, found 367.0152.

3-甲硫基亚甲基-2,2-二氟-6-溴庚酸乙酯(3ea)：44 $\mathrm{mg}$, 黄色液体, 产率 $67 \% .{ }^{1} \mathrm{H} \mathrm{NMR}\left(600 \mathrm{MHz}, \mathrm{CDCl}_{3}\right) \delta$ : 6.35 (s, 1H), 4.35 (q, J=7.1 Hz, 2H), $4.15 \sim 4.09$ (m, 1H), $2.54 \sim 2.49(\mathrm{~m}, 1 \mathrm{H}), 2.40 \sim 2.35(\mathrm{~m}, 1 \mathrm{H}), 2.31(\mathrm{~s}, 3 \mathrm{H})$, $2.00 \sim 1.88(\mathrm{~m}, 2 \mathrm{H}), 1.74(\mathrm{~d}, J=6.7 \mathrm{~Hz}, 3 \mathrm{H}), 1.36(\mathrm{t}, J=$ $7.1 \mathrm{~Hz}, 3 \mathrm{H}) ;{ }^{13} \mathrm{C}$ NMR (151 MHz, $\left.\mathrm{CDCl}_{3}\right) \delta: 163.4(\mathrm{t}, J=$ $35.0 \mathrm{~Hz}), 134.6(\mathrm{t}, J=5.9 \mathrm{~Hz}), 127.4(\mathrm{t}, J=23.6 \mathrm{~Hz})$, $113.8(\mathrm{t}, J=252.2 \mathrm{~Hz}), 63.0,50.7,39.8,31.3(\mathrm{t}, J=3.5$ $\mathrm{Hz}), 26.4,18.4,13.9 ;{ }^{19} \mathrm{~F} \mathrm{NMR}\left(565 \mathrm{MHz}, \mathrm{CDCl}_{3}\right) \delta$ : $-100.8\left(\mathrm{~d}, J=4.9 \mathrm{~Hz}\right.$ ); HRMS (ESI) calcd for $\mathrm{C}_{11} \mathrm{H}_{17} \mathrm{Br}-$ $\mathrm{F}_{2} \mathrm{O}_{2} \mathrm{SNa}(\mathrm{M}+\mathrm{Na})^{+}$352.9993, found 352.9990.

3-苯硫基亚甲基-2,2-二氟-6-溴辛酸乙酯(3fa)：65 $\mathrm{mg}$, 黄色液体, 产率 $80 \% .{ }^{1} \mathrm{H} \mathrm{NMR}\left(400 \mathrm{MHz}, \mathrm{CDCl}_{3}\right) \delta$ : $7.35 \sim 7.31(\mathrm{~m}, 4 \mathrm{H}), 7.31 \sim 7.27(\mathrm{~m}, 1 \mathrm{H}), 6.61(\mathrm{~s}, 1 \mathrm{H})$, $4.33(\mathrm{q}, J=7.1 \mathrm{~Hz}, 2 \mathrm{H}), 4.04 \sim 3.97(\mathrm{~m}, 1 \mathrm{H}), 2.68 \sim 2.61$ $(\mathrm{m}, 1 \mathrm{H}), 2.52 \sim 2.44(\mathrm{~m}, 1 \mathrm{H}), 2.09 \sim 1.92(\mathrm{~m}, 2 \mathrm{H}), 1.93 \sim$ $1.84(\mathrm{~m}, 2 \mathrm{H}), 1.33(\mathrm{t}, J=7.1 \mathrm{~Hz}, 3 \mathrm{H}), 1.06(\mathrm{t}, J=7.3 \mathrm{~Hz}$, $3 \mathrm{H}) ;{ }^{13} \mathrm{C}$ NMR $\left(151 \mathrm{MHz}, \mathrm{CDCl}_{3}\right) \delta: 163.4(\mathrm{t}, J=34.7$ $\mathrm{Hz}), 135.0,131.2(\mathrm{t}, J=5.9 \mathrm{~Hz}), 130.8(\mathrm{t}, J=23.5 \mathrm{~Hz})$, 
129.9, 129.3, 127.5, $113.6(\mathrm{t}, J=251.9 \mathrm{~Hz}), 63.2,59.0$, $37.3,32.2,31.2(\mathrm{t}, J=3.3 \mathrm{~Hz}), 13.9,12.0 ;{ }^{19} \mathrm{~F}$ NMR $(565$ $\left.\mathrm{MHz}, \mathrm{CDCl}_{3}\right) \delta:-99.0(\mathrm{~d}, J=273.1 \mathrm{~Hz}),-99.5(\mathrm{~d}, J=$ $273.1 \mathrm{~Hz}$ ); HRMS (ESI) calcd for $\mathrm{C}_{17} \mathrm{H}_{21} \mathrm{BrF}_{2} \mathrm{O}_{2} \mathrm{SNa}(\mathrm{M}+$ $\mathrm{Na})^{+}$429.0306, found 429.0320.

3-苯硫基亚甲基-2,2-二氟-6-溴十二酸乙酯(3ga): 63 $\mathrm{mg}$, 黄色液体, 产率 70\%. ${ }^{1} \mathrm{H} \mathrm{NMR}\left(400 \mathrm{MHz}, \mathrm{CDCl}_{3}\right) \delta$ : $7.35 \sim 7.32(\mathrm{~m}, 4 \mathrm{H}), 7.31 \sim 7.28(\mathrm{~m}, 1 \mathrm{H}), 6.61(\mathrm{~s}, 1 \mathrm{H})$, $4.33(\mathrm{q}, J=7.1 \mathrm{~Hz}, 2 \mathrm{H}), 4.08 \sim 4.02(\mathrm{~m}, 1 \mathrm{H}), 2.68 \sim 2.61$ $(\mathrm{m}, 1 \mathrm{H}), 2.52 \sim 2.44(\mathrm{~m}, 1 \mathrm{H}), 2.09 \sim 1.76(\mathrm{~m}, 4 \mathrm{H}), 1.57 \sim$ $1.41(\mathrm{~m}, 2 \mathrm{H}), 1.35 \sim 1.26(\mathrm{~m}, 7 \mathrm{H}), 0.90(\mathrm{t}, J=6.8 \mathrm{~Hz}, 3 \mathrm{H})$; $\left.{ }^{13} \mathrm{C} \mathrm{NMR} \mathrm{(151} \mathrm{MHz,} \mathrm{CDCl}_{3}\right) \delta: 163.4(\mathrm{t}, J=34.6 \mathrm{~Hz})$, 135.0, 131.2 (t, $J=5.9 \mathrm{~Hz}), 130.9$ (t, $J=23.5 \mathrm{~Hz}), 129.9$, 129.3, 127.5, 113.6 (t, $J=251.6 \mathrm{~Hz}), 63.2,57.4,39.2,37.7$, $31.2(\mathrm{t}, J=3.3 \mathrm{~Hz}), 31.2,27.2,22.5,14.0,13.9 ;{ }^{19} \mathrm{~F}$ NMR $\left(565 \mathrm{MHz}, \mathrm{CDCl}_{3}\right) \delta$ : $-99.0(\mathrm{~d}, J=273.1 \mathrm{~Hz}),-99.5(\mathrm{~d}$, $J=273.3 \mathrm{~Hz}$ ); HRMS (ESI) calcd for $\mathrm{C}_{20} \mathrm{H}_{27} \mathrm{BrF}_{2} \mathrm{O}_{2} \mathrm{SNa}$ $(\mathrm{M}+\mathrm{Na})^{+}$471.0775, found 471.0791 .

3-苯硫基亚甲基-8-叔丁基二甲基硅氧基-2,2-二 氟-6-溴辛酸乙酯(3ha): $60 \mathrm{mg}$, 黄色液体, 产率 $56 \% .{ }^{1} \mathrm{H}$ NMR (400 MHz, $\left.\mathrm{CDCl}_{3}\right) \delta: 7.35 \sim 7.34(\mathrm{~m}, 4 \mathrm{H}), 7.31 \sim$ $7.28(\mathrm{~m}, 1 \mathrm{H}), 6.60(\mathrm{~s}, 1 \mathrm{H}), 4.33(\mathrm{q}, J=7.1 \mathrm{~Hz}, 2 \mathrm{H}), 4.27 \sim$ $4.20(\mathrm{~m}, 1 \mathrm{H}), 3.81 \sim 3.78(\mathrm{~m}, 2 \mathrm{H}), 2.69 \sim 2.61(\mathrm{~m}, 1 \mathrm{H})$, $2.53 \sim 2.45(\mathrm{~m}, 1 \mathrm{H}), 2.10 \sim 1.95(\mathrm{~m}, 4 \mathrm{H}), 1.33(\mathrm{t}, J=7.1$ $\mathrm{Hz}, 3 \mathrm{H}), 0.89$ (s, 9H), 0.06 (d, $J=1.9 \mathrm{~Hz}, 6 \mathrm{H}) ;{ }^{13} \mathrm{C} \mathrm{NMR}$ $\left(151 \mathrm{MHz}, \mathrm{CDCl}_{3}\right) \delta: 163.4(\mathrm{t}, J=34.7 \mathrm{~Hz}), 135.1,131.2$ (t, $J=5.9 \mathrm{~Hz}), 130.7$ (t, $J=23.4 \mathrm{~Hz}), 130.0,129.3,127.6$, $113.6(\mathrm{t}, J=252.0 \mathrm{~Hz}), 63.2,60.6,53.7,41.9,38.1,31.1$ (t, $J=3.5 \mathrm{~Hz}), 25.9,18.3,13.9,-5.4,-5.4 ;{ }^{19} \mathrm{~F}$ NMR $(565$ $\left.\mathrm{MHz}, \mathrm{CDCl}_{3}\right) \delta:-99.1(\mathrm{~d}, J=272.6 \mathrm{~Hz}),-99.7(\mathrm{~d}, J=$ $272.3 \mathrm{~Hz}$ ); HRMS (ESI) calcd for $\mathrm{C}_{23} \mathrm{H}_{35} \mathrm{BrF}_{2} \mathrm{O}_{3} \mathrm{SSiNa}$ $(\mathrm{M}+\mathrm{Na})^{+}$559.1120, found 559.1129.

3-苯硫基亚甲基-8-乙酰氧基-2,2-二氟-6-溴辛酸乙 酯(3ia): $45 \mathrm{mg}$, 黄色液体, 产率 48\%. ${ }^{1} \mathrm{H}$ NMR (600 $\left.\mathrm{MHz}, \mathrm{CDCl}_{3}\right) \delta: 7.35(\mathrm{~d}, J=4.3 \mathrm{~Hz}, 4 \mathrm{H}), 7.32 \sim 7.28(\mathrm{~m}$, $1 \mathrm{H}), 6.62(\mathrm{~s}, 1 \mathrm{H}), 4.35 \sim 4.29(\mathrm{~m}, 3 \mathrm{H}), 4.25 \sim 4.21(\mathrm{~m}$, $1 \mathrm{H}), 4.16 \sim 4.12(\mathrm{~m}, 1 \mathrm{H}), 2.69 \sim 2.64(\mathrm{~m}, 1 \mathrm{H}), 2.53 \sim 2.48$ $(\mathrm{m}, 1 \mathrm{H}), 2.20 \sim 2.09(\mathrm{~m}, 3 \mathrm{H}), 2.05(\mathrm{~s}, 3 \mathrm{H}), 2.03 \sim 1.97(\mathrm{~m}$, $1 \mathrm{H}), 1.33(\mathrm{t}, J=7.1 \mathrm{~Hz}, 3 \mathrm{H}) ;{ }^{13} \mathrm{C} \mathrm{NMR}\left(151 \mathrm{MHz}, \mathrm{CDCl}_{3}\right)$ $\delta: 170.9,163.4(\mathrm{t}, J=34.7 \mathrm{~Hz}), 134.9,131.6(\mathrm{t}, J=5.8$ $\mathrm{Hz}), 130.5$ (t, $J=23.4 \mathrm{~Hz}), 123.0,129.3,127.6,113.6$ (t, $J=252.2 \mathrm{~Hz}), 63.2,62.2,52.3,37.8,37.8,31.1(\mathrm{t}, J=3.4$ $\mathrm{Hz}), 20.9,13.9 ;{ }^{19} \mathrm{~F}$ NMR $\left(565 \mathrm{MHz}, \mathrm{CDCl}_{3}\right) \delta$ : $-98.8(\mathrm{~d}$, $J=273.5 \mathrm{~Hz}),-99.7(\mathrm{~d}, J=273.4 \mathrm{~Hz})$; HRMS (ESI) calcd for $\mathrm{C}_{19} \mathrm{H}_{23} \mathrm{BrF}_{2} \mathrm{O}_{4} \mathrm{SNa}(\mathrm{M}+\mathrm{Na})^{+}$487.0361, found
487.0366.

3-苯硫基亚甲基-2,2-二氟-6,8-二溴辛酸乙酯(3ja): $62 \mathrm{mg}$, 黄色液体, 产率 64\%. ${ }^{1} \mathrm{H}$ NMR (600 MHz, $\left.\mathrm{CDCl}_{3}\right) \delta: 7.37 \sim 7.34(\mathrm{~m}, 4 \mathrm{H}), 7.32 \sim 7.29(\mathrm{~m}, 1 \mathrm{H}), 6.63$ $(\mathrm{s}, 1 \mathrm{H}), 4.35$ (q, $J=7.1 \mathrm{~Hz}, 2 \mathrm{H}), 4.28 \sim 4.23(\mathrm{~m}, 1 \mathrm{H})$, $3.64 \sim 3.61(\mathrm{~m}, 2 \mathrm{H}), 2.69 \sim 2.65(\mathrm{~m}, 1 \mathrm{H}), 2.39 \sim 2.26(\mathrm{~m}$, $2 \mathrm{H}), 2.12 \sim 2.00(\mathrm{~m}, 2 \mathrm{H}), 1.34(\mathrm{t}, J=7.1 \mathrm{~Hz}, 3 \mathrm{H}) ;{ }^{13} \mathrm{C}$ NMR (151 MHz, $\left.\mathrm{CDCl}_{3}\right) \delta: 163.4(\mathrm{t}, J=34.6 \mathrm{~Hz}), 134.8$, $131.7(\mathrm{t}, J=5.9 \mathrm{~Hz}), 130.6(\mathrm{t}, J=11.7 \mathrm{~Hz}), 130.1,129.3$, 127.6, 113.5 (t, $J=251.4 \mathrm{~Hz}$ ), 63.3, 54.0, 41.3, 37.5, 31.1, $31.0(\mathrm{t}, J=3.4 \mathrm{~Hz}), 13.9 ;{ }^{19} \mathrm{~F}$ NMR $\left(565 \mathrm{MHz} \mathrm{CDCl}_{3}\right) \delta$ : $-98.5(\mathrm{~d}, J=273.4 \mathrm{~Hz}),-99.8(\mathrm{~d}, J=273.3 \mathrm{~Hz})$; HRMS (ESI) calcd for $\mathrm{C}_{17} \mathrm{H}_{20} \mathrm{Br}_{2} \mathrm{~F}_{2} \mathrm{O}_{2} \mathrm{SNa}(\mathrm{M}+\mathrm{Na})^{+}$506.9411, found 506.9413 .

3-苯硫基亚甲基-2,2-二氟-8-氯-6-溴辛酸乙酯(3ka): $48 \mathrm{mg}$, 黄色液体, 产率 55\%. ${ }^{1} \mathrm{H}$ NMR (400 MHz, $\left.\mathrm{CDCl}_{3}\right) \delta: 7.38 \sim 7.30(\mathrm{~m}, 5 \mathrm{H}), 6.64(\mathrm{~s}, 1 \mathrm{H}), 4.36(\mathrm{q}, J=$ $7.1 \mathrm{~Hz}, 2 \mathrm{H}), 4.22 \sim 4.26(\mathrm{~m}, 1 \mathrm{H}), 3.84 \sim 3.74(\mathrm{~m}, 2 \mathrm{H})$, $2.72 \sim 2.65(\mathrm{~m}, 1 \mathrm{H}), 2.58 \sim 2.50(\mathrm{~m}, 1 \mathrm{H}), 2.34 \sim 2.19(\mathrm{~m}$, $2 \mathrm{H}), 2.15 \sim 1.99(\mathrm{~m}, 2 \mathrm{H}), 1.35(\mathrm{t}, J=7.1 \mathrm{~Hz}, 3 \mathrm{H}) ;{ }^{13} \mathrm{C}$ NMR (151 MHz, $\left.\mathrm{CDCl}_{3}\right) \delta: 163.4(\mathrm{t}, J=34.6 \mathrm{~Hz}), 134.9$, $131.7(\mathrm{t}, J=5.9 \mathrm{~Hz}), 130.6(\mathrm{t}, J=23.9 \mathrm{~Hz}), 130.1,129.3$, 127.6, 113.5 (t, $J=252.6 \mathrm{~Hz}), 63.3,52.8,42.7,41.3,37.6$, $31.0(\mathrm{t}, J=3.6 \mathrm{~Hz}), 13.9 ;{ }^{19} \mathrm{~F}$ NMR (565 MHz, $\left.\mathrm{CDCl}_{3}\right) \delta$ : $-98.5(\mathrm{~d}, J=273.6 \mathrm{~Hz}),-99.9$ (d, $J=273.5 \mathrm{~Hz})$; HRMS (ESI) calcd for $\mathrm{C}_{17} \mathrm{H}_{20} \mathrm{BrClF}_{2} \mathrm{O}_{2} \mathrm{SNa}(\mathrm{M}+\mathrm{Na})^{+} 462.9916$, found 462.9921 .

3-苯硫基亚甲基-8-邻苯二甲酰胺基-2,2-二氟-6-溴 辛酸乙酯(3la): $56 \mathrm{mg}$, 黄色液体, 产率 $51 \%$. ${ }^{1} \mathrm{H} \mathrm{NMR}$ $\left(600 \mathrm{MHz}, \mathrm{CDCl}_{3}\right) \delta: 7.85 \sim 7.82(\mathrm{~m}, 2 \mathrm{H}), 7.73 \sim 7.70(\mathrm{~m}$, $2 \mathrm{H}), 7.38 \sim 7.33(\mathrm{~m}, 4 \mathrm{H}), 7.31 \sim 7.29(\mathrm{~m}, 1 \mathrm{H}), 6.61(\mathrm{~s}$, $1 \mathrm{H}), 4.27 \sim 4.22(\mathrm{~m}, 2 \mathrm{H}), 4.08 \sim 4.05(\mathrm{~m}, 1 \mathrm{H}), 3.96 \sim 3.87$ $(\mathrm{m}, 2 \mathrm{H}), 2.67 \sim 2.63(\mathrm{~m}, 1 \mathrm{H}), 2.52 \sim 2.47(\mathrm{~m}, 1 \mathrm{H}), 2.31 \sim$ $2.19(\mathrm{~m}, 2 \mathrm{H}), 2.11 \sim 1.98(\mathrm{~m}, 2 \mathrm{H}), 1.29(\mathrm{t}, J=7.1 \mathrm{~Hz}, 3 \mathrm{H})$; ${ }^{13} \mathrm{C}$ NMR $\left(151 \mathrm{MHz}, \mathrm{CDCl}_{3}\right) \delta: 168.2,163.4(\mathrm{t}, J=34.6$ $\mathrm{Hz}), 134.9,134.0,132.0,131.6(\mathrm{t}, J=5.7 \mathrm{~Hz}), 130.5(\mathrm{t}$, $J=23.1 \mathrm{~Hz}), 130.0,129.3,127.6,123.3,113.5$ (t, $J=252.2$ $\mathrm{Hz}), 63.1,52.8,37.7,37.6,36.4,31.0$ (t, $J=3.3 \mathrm{~Hz}), 13.9$; ${ }^{19} \mathrm{~F}$ NMR $\left(565 \mathrm{MHz}, \mathrm{CDCl}_{3}\right) \delta:-98.2(\mathrm{~d}, J=274.0 \mathrm{~Hz})$, -100.2 (d, $J=274.0 \mathrm{~Hz}$ ); HRMS (ESI) calcd for $\mathrm{C}_{25} \mathrm{H}_{24}{ }^{-}$ $\mathrm{BrF}_{2} \mathrm{NO}_{4} \mathrm{SNa}(\mathrm{M}+\mathrm{Na})^{+}$574.0470, found 574.0469.

3-甲硫基亚甲基-8-甲氧基-2,2-二氟-6-溴辛酸乙酯 (3ma): $46 \mathrm{mg}$, 黄色液体, 产率 62\%. ${ }^{1} \mathrm{H} \mathrm{NMR}(600 \mathrm{MHz}$, $\left.\mathrm{CDCl}_{3}\right) \delta: 6.33(\mathrm{~s}, 1 \mathrm{H}), 4.34(\mathrm{q}, J=7.1 \mathrm{~Hz}, 2 \mathrm{H}), 4.18 \sim$ $4.14(\mathrm{~m}, 1 \mathrm{H}), 3.58 \sim 3.51(\mathrm{~m}, 2 \mathrm{H}), 3.34(\mathrm{~s}, 3 \mathrm{H}), 2.57 \sim$ 
$2.52(\mathrm{~m}, 1 \mathrm{H}), 2.40 \sim 2.35(\mathrm{~m}, 1 \mathrm{H}), 2.29(\mathrm{~s}, 3 \mathrm{H}), 2.12 \sim$ $2.07(\mathrm{~m}, 1 \mathrm{H}), 2.05 \sim 2.00(\mathrm{~m}, 2 \mathrm{H}), 1.97 \sim 1.91(\mathrm{~m}, 1 \mathrm{H})$, $1.35(\mathrm{t}, J=7.1 \mathrm{~Hz}, 3 \mathrm{H}) ;{ }^{13} \mathrm{C} \mathrm{NMR}\left(151 \mathrm{MHz}, \mathrm{CDCl}_{3}\right) \delta$ : 163.4 (t, $J=35.0 \mathrm{~Hz}), 134.6$ (t, $J=5.8 \mathrm{~Hz}), 127.5$ (t, $J=$ $23.6 \mathrm{~Hz}), 113.8(\mathrm{t}, J=252.1 \mathrm{~Hz}), 70.1,63.0,58.8,53.7$, $39.1,38.3,31.1(\mathrm{t}, J=3.5 \mathrm{~Hz}), 18.4,13.9 ;{ }^{19} \mathrm{~F}$ NMR (565 $\mathrm{MHz}, \mathrm{CDCl}_{3}$ ) $\delta$ : -100.8 ; HRMS (ESI) calcd for $\mathrm{C}_{13} \mathrm{H}_{21}$ $\mathrm{BrF}_{2} \mathrm{O}_{3} \mathrm{SNa}(\mathrm{M}+\mathrm{Na})^{+}$397.0255, found 397.0258.

3-甲硫基亚甲基-4-(2'-溴环己基)-2,2-二氟丁酸乙酯 (3na): $62 \mathrm{mg}$, 黄色液体, 产率 $84 \%, d r 1.8: 1 .{ }^{1} \mathrm{H}$ NMR $\left(400 \mathrm{MHz}, \mathrm{CDCl}_{3}\right)$ of major isomer $\delta: 6.48(\mathrm{~s}, 1 \mathrm{H}), 4.56(\mathrm{~s}$, $1 \mathrm{H}), 4.34$ (q, $J=7.1 \mathrm{~Hz}, 2 \mathrm{H}), 2.30(\mathrm{~s}, 3 \mathrm{H}), 2.24(\mathrm{~m}, 1 \mathrm{H})$, $2.17(\mathrm{~d}, J=13.9 \mathrm{~Hz}, 1 \mathrm{H}), 1.87 \sim 1.78(\mathrm{~m}, 2 \mathrm{H}), 1.77 \sim 1.73$ $(\mathrm{m}, 1 \mathrm{H}), 1.58 \sim 1.52(\mathrm{~m}, 2 \mathrm{H}), 1.48 \sim 1.44(\mathrm{~m}, 1 \mathrm{H}), 1.35(\mathrm{t}$, $J=7.1 \mathrm{~Hz}, 3 \mathrm{H}), 1.33 \sim 1.28(\mathrm{~m}, 1 \mathrm{H}), 1.27 \sim 1.25(\mathrm{~m}, 1 \mathrm{H})$; ${ }^{13} \mathrm{C}$ NMR (151 MHz, $\mathrm{CDCl}_{3}$ ) of major isomer $\delta: 163.5(\mathrm{t}$, $J=35.1 \mathrm{~Hz}), 135.8(\mathrm{t}, J=6.3 \mathrm{~Hz}), 125.9$ (t, $J=23.6 \mathrm{~Hz})$, $113.8(\mathrm{t}, J=251.8 \mathrm{~Hz}), 63.0,61.7,40.8,39.1,35.0,27.0$, $25.5,20.8,18.3,13.9 ;{ }^{19} \mathrm{~F}$ NMR $\left(565 \mathrm{MHz}, \mathrm{CDCl}_{3}\right)$ of major isomer $\delta$ : $-99.8(\mathrm{~d}, J=268.8 \mathrm{~Hz}),-100.5(\mathrm{~d}, J=$ $268.8 \mathrm{~Hz}$ ); HRMS (ESI) calcd for $\mathrm{C}_{14} \mathrm{H}_{21} \mathrm{BrF}_{2} \mathrm{O}_{2} \mathrm{SNa}(\mathrm{M}+$ $\mathrm{Na})^{+}$393.0306, found 393.0304.

3-甲硫基亚甲基-4-甲氧基-2,2-二氟-6-溴辛酸乙酯 (3oa): $47 \mathrm{mg}$, 黄色液体, 产率 63\%, $d r 1.5: 1 .{ }^{1} \mathrm{H} \mathrm{NMR}$ $\left(400 \mathrm{MHz}, \mathrm{CDCl}_{3}\right)$ of major isomer $\delta: 6.62(\mathrm{~s}, 1 \mathrm{H}), 4.39 \sim$ $4.32(\mathrm{~m}, 2 \mathrm{H}), 4.28 \sim 4.22(\mathrm{~m}, 1 \mathrm{H}), 4.18(\mathrm{~d}, J=9.7 \mathrm{~Hz} 1 \mathrm{H})$, $3.30(\mathrm{~s}, 3 \mathrm{H}), 2.37(\mathrm{~s}, 3 \mathrm{H}), 2.27 \sim 2.16(\mathrm{~m}, 1 \mathrm{H}), 2.07 \sim 1.79$ (m, 3H), 1.37 (t, $J=7.2 \mathrm{~Hz}, 3 \mathrm{H}), 1.06(\mathrm{t}, J=7.2 \mathrm{~Hz}, 3 \mathrm{H})$; ${ }^{13} \mathrm{C}$ NMR $\left(151 \mathrm{MHz}, \mathrm{CDCl}_{3}\right.$ ) of major isomer $\delta: 163.1(\mathrm{t}$, $J=34.9 \mathrm{~Hz}), 136.2(\mathrm{t}, J=5.5 \mathrm{~Hz}), 127.1(\mathrm{t}, J=22.7 \mathrm{~Hz})$, 113.6 (t, $J=252.3 \mathrm{~Hz}), 78.7$ (t, $J=3.6 \mathrm{~Hz}), 57.0,56.6$, 46.2, 32.6, 18.7, 13.9, 11.9; ${ }^{19} \mathrm{~F}$ NMR $\left(565 \mathrm{MHz}, \mathrm{CDCl}_{3}\right)$ of major isomer $\delta$ : $-99.4(\mathrm{~d}, J=270.2 \mathrm{~Hz}),-103.18$ (d, $J=270.1 \mathrm{~Hz}$ ); HRMS (ESI) calcd for $\mathrm{C}_{13} \mathrm{H}_{21} \mathrm{BrF}_{2} \mathrm{O}_{3} \mathrm{SNa}$ $(\mathrm{M}+\mathrm{Na})^{+}$397.0255, found 397.0268.

6-甲基-3-甲硫基亚甲基-2,2-二氟-6-溴庚酸乙酯 (3pa): $38 \mathrm{mg}$, 黄色液体, 产率 55\%. ${ }^{1} \mathrm{H}$ NMR $(400 \mathrm{MHz}$, $\left.\mathrm{CDCl}_{3}\right) \delta: 6.30(\mathrm{~s}, 1 \mathrm{H}), 4.35(\mathrm{q}, J=7.1 \mathrm{~Hz}, 2 \mathrm{H}), 2.49 \sim$ $2.45(\mathrm{~m}, 2 \mathrm{H}), 2.30(\mathrm{~s}, 3 \mathrm{H}), 1.97 \sim 1.93(\mathrm{~m}, 2 \mathrm{H}), 1.77(\mathrm{~s}$, $6 \mathrm{H}), 1.36(\mathrm{t}, J=7.1 \mathrm{~Hz}, 3 \mathrm{H}) ;{ }^{13} \mathrm{C} \mathrm{NMR}\left(151 \mathrm{MHz}, \mathrm{CDCl}_{3}\right)$ $\delta: 163.4(\mathrm{t}, J=35.2 \mathrm{~Hz}), 133.9$ (t, $J=5.7 \mathrm{~Hz}), 128.3(\mathrm{t}, J=$ $23.2 \mathrm{~Hz}), 113.9$ (t, $J=252.2 \mathrm{~Hz}), 66.9,63.0,46.7,34.1$, 30.0 (t, $J=3.7 \mathrm{~Hz}), 18.4,13.9 ;{ }^{19} \mathrm{~F}$ NMR $(565 \mathrm{MHz}$, $\mathrm{CDCl}_{3}$ ) $\delta$ : -100.9 ; HRMS (ESI) calcd for $\mathrm{C}_{12} \mathrm{H}_{19} \mathrm{BrF}_{2}$ $\mathrm{O}_{2} \mathrm{SNa}(\mathrm{M}+\mathrm{Na})^{+}$367.0149, found 367.0145.
5,5-二甲基-3-苯硫基亚甲基-2,2-二氟-6-溴己酸乙 酯(3qa): $30 \mathrm{mg}$, 黄色液体, 产率 37\%; ${ }^{1} \mathrm{H}$ NMR (400 $\left.\mathrm{MHz}, \mathrm{CDCl}_{3}\right) \delta: 7.37 \sim 7.28(\mathrm{~m}, 5 \mathrm{H}), 6.72(\mathrm{~s}, 1 \mathrm{H}), 4.31(\mathrm{q}$, $J=7.1 \mathrm{~Hz}, 2 \mathrm{H}), 3.36$ (s, 2H), 2.45 (s, 2H), 1.31 (t, $J=7.1$ $\mathrm{Hz}, 3 \mathrm{H}), 1.08$ (s, 6H); ${ }^{13} \mathrm{C}$ NMR (151 MHz, $\left.\mathrm{CDCl}_{3}\right) \delta$ : $163.6(\mathrm{t}, J=35.2 \mathrm{~Hz}), 134.7,133.6(\mathrm{t}, J=6.6 \mathrm{~Hz}), 130.4$, 129.7, 129.3, 127.5, 113.0 (t, $J=251.6 \mathrm{~Hz}), 63.1,46.1$, $40.5(\mathrm{t}, J=2.3 \mathrm{~Hz}), 36.2,25.9,13.9 ;{ }^{19} \mathrm{~F}$ NMR $(565 \mathrm{MHz}$, $\left.\mathrm{CDCl}_{3}\right) \delta$ : -96.1 ; HRMS (ESI) calcd for $\mathrm{C}_{17} \mathrm{H}_{21} \mathrm{BrF}_{2}-$ $\mathrm{O}_{2} \mathrm{SNa}(\mathrm{M}+\mathrm{Na})^{+}$429.0306, found 429.0310 .

6-苯基-3-甲硫基亚甲基-2,2-二氟-6-溴己酸乙酯 (3ra): $49 \mathrm{mg}$, 黄色液体, 产率 62\%. ${ }^{1} \mathrm{H}$ NMR (400 MHz, $\left.\mathrm{CDCl}_{3}\right) \delta: 7.41 \sim 7.39(\mathrm{~m}, 2 \mathrm{H}), 7.37 \sim 7.33(\mathrm{~m}, 2 \mathrm{H})$, $7.32 \sim 7.28(\mathrm{~m}, 1 \mathrm{H}), 6.31(\mathrm{~s}, 1 \mathrm{H}), 5.00 \sim 4.95(\mathrm{~m}, 1 \mathrm{H})$, 4.33 (q, $J=7.1 \mathrm{~Hz}, 2 \mathrm{H}), 2.51 \sim 2.33(\mathrm{~m}, 4 \mathrm{H}), 2.30(\mathrm{~s}, 3 \mathrm{H})$, $1.34(\mathrm{t}, J=7.1 \mathrm{~Hz}, 3 \mathrm{H}) ;{ }^{13} \mathrm{C}$ NMR $\left(151 \mathrm{MHz}, \mathrm{CDCl}_{3}\right) \delta$ : $163.4(\mathrm{t}, J=35.0 \mathrm{~Hz}), 141.6,134.9(\mathrm{t}, J=5.9 \mathrm{~Hz}), 128.8$, $128.5,127.3,127.0(\mathrm{t}, J=23.9 \mathrm{~Hz}), 113.7(\mathrm{t}, J=252.4$ $\mathrm{Hz}), 63.1,54.5,38.5,31.7$ (t, $J=3.4 \mathrm{~Hz}), 18.4,13.9 ;{ }^{19} \mathrm{~F}$ NMR $\left(565 \mathrm{MHz}, \mathrm{CDCl}_{3}\right) \delta$ : $-100.5(\mathrm{~d}, J=269.6 \mathrm{~Hz})$, -101.0 (d, $J=269.7 \mathrm{~Hz}$ ); HRMS (ESI) calcd for $\mathrm{C}_{16} \mathrm{H}_{19} \mathrm{BrF}_{2} \mathrm{O}_{2} \mathrm{SNa}(\mathrm{M}+\mathrm{Na})^{+}$415.0149, found 415.0150.

7-苯基-3-甲硫基亚甲基-2,2-二氟-6-溴庚酸乙酯 (3sa): $40 \mathrm{mg}$, 黄色液体, 产率 50\%. ${ }^{1} \mathrm{H}$ NMR $(600 \mathrm{MHz}$, $\left.\mathrm{CDCl}_{3}\right) \delta: 7.34 \sim 7.28(\mathrm{~m}, 3 \mathrm{H}), 7.24 \sim 7.21(\mathrm{~m}, 2 \mathrm{H}), 6.29$ (s, 1H), 4.33 (q, $J=7.1 \mathrm{~Hz}, 1 \mathrm{H}), 4.22 \sim 4.17(\mathrm{~m}, 1 \mathrm{H})$, $3.24 \sim 3.14(\mathrm{~m}, 1 \mathrm{H}), 2.62 \sim 2.55(\mathrm{~m}, 1 \mathrm{H}), 2.45 \sim 2.35(\mathrm{~m}$, $1 \mathrm{H}), 2.28(\mathrm{~s}, 3 \mathrm{H}), 2.11 \sim 2.02(\mathrm{~m}, 1 \mathrm{H}), 1.95 \sim 1.86(\mathrm{~m}$, $1 \mathrm{H}), 1.35(\mathrm{t}, J=7.2 \mathrm{~Hz}, 2 \mathrm{H}) ;{ }^{13} \mathrm{C} \mathrm{NMR}\left(151 \mathrm{MHz}, \mathrm{CDCl}_{3}\right)$ $\delta$ : $163.4(\mathrm{t}, J=34.7 \mathrm{~Hz}), 138.1,134.7(\mathrm{t}, J=5.7 \mathrm{~Hz})$, 129.2, 128.4, $127.2(\mathrm{t}, J=23.6 \mathrm{~Hz}), 126.9,113.8(\mathrm{t}, J=$ $252.3 \mathrm{~Hz}), 63.0,56.5,45.6,37.1,31.0$ (t, $J=3.4 \mathrm{~Hz}), 18.4$, 13.9; ${ }^{19} \mathrm{~F}$ NMR $\left(565 \mathrm{MHz}, \mathrm{CDCl}_{3}\right) \delta:-100.5(\mathrm{~d}, J=$ $268.7 \mathrm{~Hz}$ ), $-101.1(\mathrm{~d}, J=268.7 \mathrm{~Hz}$ ); HRMS (ESI) calcd for $\mathrm{C}_{17} \mathrm{H}_{21} \mathrm{BrF}_{2} \mathrm{O}_{2} \mathrm{SNa} \quad(\mathrm{M}+\mathrm{Na})^{+} 429.0306$, found 429.0309 .

3-甲硒基亚甲基-2,2-二氟-6-溴庚酸乙酯(3ta)：28 $\mathrm{mg}$, 黄色液体, 产率 $37 \% .{ }^{1} \mathrm{H} \mathrm{NMR}\left(400 \mathrm{MHz}, \mathrm{CDCl}_{3}\right) \delta$ : 6.68 (s, 1H), 4.35 (q, $J=7.1 \mathrm{~Hz}, 2 \mathrm{H}), 4.15 \sim 4.07(\mathrm{~m}, 1 \mathrm{H})$, $2.53 \sim 2.46(\mathrm{~m}, 1 \mathrm{H}), 2.41 \sim 2.33(\mathrm{~m}, 1 \mathrm{H}), 2.18(\mathrm{~s}, 3 \mathrm{H})$, $2.02 \sim 1.87(\mathrm{~m}, 2 \mathrm{H}), 1.73(\mathrm{~d}, J=6.7 \mathrm{~Hz}, 3 \mathrm{H}), 1.36(\mathrm{t}, J=$ $7.1 \mathrm{~Hz}, 3 \mathrm{H}) ;{ }^{13} \mathrm{C}$ NMR $\left(151 \mathrm{MHz}, \mathrm{CDCl}_{3}\right) \delta: 163.2(\mathrm{t}, J=$ $35.2 \mathrm{~Hz}), 130.1(\mathrm{t}, J=23.0 \mathrm{~Hz}), 129.5(\mathrm{t}, J=5.8 \mathrm{~Hz})$, 114.4 (t, $J=253.2 \mathrm{~Hz}), 63.2,50.7,39.7,32.1$ (t, $J=3.4$ $\mathrm{Hz}), 26.5,14.0,8.6 ;{ }^{19} \mathrm{~F}$ NMR (565 $\left.\mathrm{MHz}, \mathrm{CDCl}_{3}\right) \delta$ : 
$-101.7(\mathrm{~d}, J=262.5 \mathrm{~Hz}),-102.2(\mathrm{~d}, J=262.5 \mathrm{~Hz})$; HRMS (ESI) calcd for $\mathrm{C}_{11} \mathrm{H}_{17} \mathrm{~F}_{2} \mathrm{O}_{2} \mathrm{SeNa}(\mathrm{M}+\mathrm{Na})^{+}$ 400.9437, found 400.9438 .

3-苯硫基亚甲基-1-对甲苯磺酰哌嗪基-2,2-二氟-6澳庚酮(3ab): $83 \mathrm{mg}$, 黄色液体, 产率 $71 \% .{ }^{1} \mathrm{H}$ NMR $\left(600 \mathrm{MHz}, \mathrm{CDCl}_{3}\right) \delta: 7.63(\mathrm{~d}, J=8.2 \mathrm{~Hz}, 1 \mathrm{H}), 7.37 \sim 7.31$ (m, 3H), $6.55(\mathrm{~s}, 1 \mathrm{H}), 4.11 \sim 4.09(\mathrm{~m}, 1 \mathrm{H}), 3.80 \sim 3.68(\mathrm{~m}$, $4 \mathrm{H}), 3.07 \sim 3.05(\mathrm{~m}, 4 \mathrm{H}), 2.46 \sim 2.41(\mathrm{~m}, 4 \mathrm{H}), 2.34 \sim 2.29$ $(\mathrm{m}, 1 \mathrm{H}), 1.97 \sim 1.84(\mathrm{~m}, 2 \mathrm{H}), 1.70(\mathrm{~d}, J=6.7 \mathrm{~Hz}, 2 \mathrm{H}) ;{ }^{13} \mathrm{C}$ NMR (151 MHz, $\left.\mathrm{CDCl}_{3}\right) \delta: 161.2(\mathrm{t}, J=30.9 \mathrm{~Hz}), 144.2$, 134.9 (t, $J=2.4 \mathrm{~Hz}), 132.1,131.8(\mathrm{t}, J=4.9 \mathrm{~Hz}), 130.5$, 129.9, 129.4, 127.9, 127.7, 116.3 (t, $J=253.7 \mathrm{~Hz}), 50.6$, 46.0, 45.7, 45.3, 42.6, 39.6, 31.3 (t, $J=3.0 \mathrm{~Hz}), 26.4,21.6$; ${ }^{19} \mathrm{~F}$ NMR $(565 \mathrm{MHz}, \mathrm{CDCl} 3) \delta:-93.6(\mathrm{~d}, J=279.0 \mathrm{~Hz})$, -94.1 (d, $J=278.9 \mathrm{~Hz}$ ); HRMS (ESI) calcd for $\mathrm{C}_{25} \mathrm{H}_{29} \mathrm{Br}-$ $\mathrm{F}_{2} \mathrm{~N}_{2} \mathrm{O}_{3} \mathrm{~S}_{2} \mathrm{Na}(\mathrm{M}+\mathrm{Na})^{+}$609.0663, found 609.0657.

3-苯硫基亚甲基-1-叔丁氧羰基哌嗪基-2,2-二氟-6溴庚酮(3ac): $79 \mathrm{mg}$, 黄色液体, 产率 74\%. ${ }^{1} \mathrm{H}$ NMR (600 $\left.\mathrm{MHz}, \mathrm{CDCl}_{3}\right) \delta: 7.40(\mathrm{~d}, J=7.3 \mathrm{~Hz}, 2 \mathrm{H}), 7.36(\mathrm{t}, J=7.5$ $\mathrm{Hz}, 2 \mathrm{H}), 7.33 \sim 7.30(\mathrm{~m}, 1 \mathrm{H}), 6.60(\mathrm{~s}, 1 \mathrm{H}), 4.18 \sim 4.12(\mathrm{~m}$, $1 \mathrm{H}), 3.63(\mathrm{~d}, J=32.8 \mathrm{~Hz}, 4 \mathrm{H}), 3.49$ (d, $J=4.5 \mathrm{~Hz}, 4 \mathrm{H})$, $2.54 \sim 2.50(\mathrm{~m}, 1 \mathrm{H}), 2.42 \sim 2.37(\mathrm{~m}, 1 \mathrm{H}), 2.00 \sim 1.99(\mathrm{~m}$, $1 \mathrm{H}), 1.94 \sim 1.92(\mathrm{~m}, 1 \mathrm{H}), 1.73(\mathrm{~d}, J=6.6 \mathrm{~Hz}, 3 \mathrm{H}), 1.47$ (s, 9H); ${ }^{13} \mathrm{C}$ NMR (151 MHz, $\left.\mathrm{CDCl}_{3}\right) \delta: 161.3$ (t, $J=31.6$ $\mathrm{Hz}), 154.4,135.2,131.6(\mathrm{t}, J=5.7 \mathrm{~Hz}), 130.5,129.4,128.4$ (t, $J=22.9 \mathrm{~Hz}), 127.8,118.2(\mathrm{t}, J=253.1 \mathrm{~Hz}), 80.5,50.7$, 45.7, 43.2, 39.7, 31.5 (t, $J=3.0 \mathrm{~Hz}), 28.3,26.5 ;{ }^{19} \mathrm{~F}$ NMR $\left(565 \mathrm{MHz}, \mathrm{CDCl}_{3}\right) \delta$ : -94.0 ; HRMS (ESI) calcd for $\mathrm{C}_{23} \mathrm{H}_{31} \mathrm{BrF}_{2} \mathrm{~N}_{2} \mathrm{O}_{3} \mathrm{SNa}(\mathrm{M}+\mathrm{Na})^{+} 555.1099$, found 555.1095 .

3-苯硫基亚甲基-1-吗啉基-2,2-二氟-6-溴庚酮(3ad): $53 \mathrm{mg}$, 黄色液体, 产率 $61 \% .{ }^{1} \mathrm{H}$ NMR $(600 \mathrm{MHz}$, $\left.\mathrm{CDCl}_{3}\right) \delta: 7.40(\mathrm{~d}, J=7.4 \mathrm{~Hz}, 2 \mathrm{H}), 7.36(\mathrm{t}, J=7.5 \mathrm{~Hz}, 2 \mathrm{H})$, 7.31 (t, $J=7.2 \mathrm{~Hz}, 1 \mathrm{H}), 6.60(\mathrm{~s}, 1 \mathrm{H}), 4.20 \sim 4.13(\mathrm{~m}, 1 \mathrm{H})$, $3.75 \sim 3.65(\mathrm{~m}, 8 \mathrm{H}), 2.55 \sim 2.50(\mathrm{~m}, 1 \mathrm{H}), 2.43 \sim 2.37(\mathrm{~m}$, $1 \mathrm{H}), 2.04 \sim 1.98(\mathrm{~m}, 1 \mathrm{H}), 1.96 \sim 1.90(\mathrm{~m}, 1 \mathrm{H}), 1.73(\mathrm{~d}, J=$ $6.6 \mathrm{~Hz}, 3 \mathrm{H}) ;{ }^{13} \mathrm{C}$ NMR $\left(151 \mathrm{MHz}, \mathrm{CDCl}_{3}\right) \delta: 161.2(\mathrm{t}, J=$ $30.4 \mathrm{~Hz}), 135.2$ (t, $J=1.9 \mathrm{~Hz}), 131.6(\mathrm{t}, J=5.1 \mathrm{~Hz}), 130.4$, 129.4, 127.8, 116.4 (t, $J=253.3 \mathrm{~Hz}), 66.7,66.5,50.7,46.5$, 43.5, 39.7, 31.5 (t, $J=3.4 \mathrm{~Hz}), 26.5 ;{ }^{19} \mathrm{~F}$ NMR $(565 \mathrm{MHz}$, $\left.\mathrm{CDCl}_{3}\right) \delta:-93.8(\mathrm{~d}, J=278.2 \mathrm{~Hz}),-94.3(\mathrm{~d}, J=278.2$ $\mathrm{Hz}$ ); HRMS (ESI) calcd for $\mathrm{C}_{18} \mathrm{H}_{22} \mathrm{BrF}_{2} \mathrm{NO}_{2} \mathrm{SNa}(\mathrm{M}+$ $\mathrm{Na})^{+}$456.0415, found 456.0411 .

3 -苯硫基亚甲基-2,2-二氟-6-溴- $N, N$-二乙基庚酰胺 (3ae): $36 \mathrm{mg}$, 黄色液体, 产率 43\%. ${ }^{1} \mathrm{H}$ NMR (400 MHz,
$\left.\mathrm{CDCl}_{3}\right) \delta: 7.41(\mathrm{~d}, J=7.4 \mathrm{~Hz}, 2 \mathrm{H}), 7.35(\mathrm{t}, J=7.4 \mathrm{~Hz}, 2 \mathrm{H})$, $7.30(\mathrm{~d}, J=7.0 \mathrm{~Hz}, 1 \mathrm{H}), 6.57(\mathrm{~s}, 1 \mathrm{H}), 4.21 \sim 4.13(\mathrm{~m}, 1 \mathrm{H})$, $3.49 \sim 3.40(\mathrm{~m}, 4 \mathrm{H}), 2.53 \sim 2.46(\mathrm{~m}, 1 \mathrm{H}), 2.42 \sim 2.32(\mathrm{~m}$, $1 \mathrm{H}), 2.06 \sim 1.89(\mathrm{~m}, 2 \mathrm{H}), 1.73(\mathrm{~d}, J=6.6 \mathrm{~Hz}, 3 \mathrm{H}), 1.24(\mathrm{t}$, $J=6.9 \mathrm{~Hz}, 3 \mathrm{H}), 1.17(\mathrm{t}, J=7.1 \mathrm{~Hz}, 3 \mathrm{H}) ;{ }^{13} \mathrm{C}$ NMR $(151$ $\left.\mathrm{MHz}, \mathrm{CDCl}_{3}\right) \delta: 161.9$ (t, $J=30.3 \mathrm{~Hz}$ ), 135.6 (t, $J=2.5$ Hz), 130.9 (t, $J=4.7 \mathrm{~Hz}), 130.5,129.3,129.2$ (t, $J=22.6$ Hz), $116.7(\mathrm{t}, J=254.1 \mathrm{~Hz}), 50.9,41.9(\mathrm{t}, J=4.1 \mathrm{~Hz})$, $41.7,39.7,31.6(\mathrm{t}, J=3.3 \mathrm{~Hz}), 26.5,14.1,12.3 ;{ }^{19} \mathrm{~F}$ NMR $\left(565 \mathrm{MHz}, \mathrm{CDCl}_{3}\right) \delta$ : $-93.6(\mathrm{~d}, J=274.4 \mathrm{~Hz}),-94.6(\mathrm{~d}$, $J=274.4 \mathrm{~Hz}$ ); HRMS (ESI) calcd for $\mathrm{C}_{18} \mathrm{H}_{24} \mathrm{BrF}_{2} \mathrm{NOSNa}$ $(\mathrm{M}+\mathrm{Na})^{+}$442.0622, found 442.0619.

3-苯硫基亚甲基-2,2-二氟-6-澳- $N$-芐基庚酰胺(3af): $59 \mathrm{mg}$, 黄色液体, 产率 $54 \% .{ }^{1} \mathrm{H}$ NMR $(400 \mathrm{MHz}$, $\left.\mathrm{CDCl}_{3}\right) \delta: 7.39 \sim 7.32(\mathrm{~m}, 5 \mathrm{H}), 7.31 \sim 7.26(\mathrm{~m}, 5 \mathrm{H}), 6.93$ (s, $1 \mathrm{H}), 6.60(\mathrm{~s}, 1 \mathrm{H}), 4.55(\mathrm{dd}, J=5.5,3.6 \mathrm{~Hz}, 2 \mathrm{H}), 4.22 \sim$ $4.13(\mathrm{~m}, 1 \mathrm{H}), 2.70 \sim 2.63(\mathrm{~m}, 1 \mathrm{H}), 2.55 \sim 2.48(\mathrm{~m}, 1 \mathrm{H})$, $2.13 \sim 2.05(\mathrm{~m}, 1 \mathrm{H}), 2.01 \sim 1.91(\mathrm{~m}, 1 \mathrm{H}), 1.76(\mathrm{~d}, J=6.6$ $\mathrm{Hz}, 3 \mathrm{H}) ;{ }^{13} \mathrm{C}$ NMR $\left(151 \mathrm{MHz}, \mathrm{CDCl}_{3}\right) \delta: 163.4(\mathrm{t}, J=30.9$ $\mathrm{Hz}), 136.8134 .8,131.1$ (t, $J=23.3 \mathrm{~Hz}), 130.6$ (t, $J=5.3$ $\mathrm{Hz}), 130.3,129.4,128.8,127.9,127.9,127.7,115.4$ (t, $J=$ $255.6 \mathrm{~Hz}), 50.9,43.8,39.5,31.8(\mathrm{t}, J=3.5 \mathrm{~Hz}), 26.5 ;{ }^{19} \mathrm{~F}$ NMR $\left(565 \mathrm{MHz}, \mathrm{CDCl}_{3}\right) \delta:-98.9(\mathrm{~d}, J=260.6 \mathrm{~Hz})$, -100.2 (d, $J=260.6 \mathrm{~Hz}$ ); HRMS (ESI) calcd for $\mathrm{C}_{21} \mathrm{H}_{22} \mathrm{BrF}_{2} \mathrm{NOSNa}(\mathrm{M}+\mathrm{Na})^{+}$476.0466, found 476.0463.

3-苯硫基亚甲基-2,2-二氟-6-溴庚酸甲酯(3ag)：57 $\mathrm{mg}$, 黄色液体, 产率 $75 \% .{ }^{1} \mathrm{H} \mathrm{NMR}\left(600 \mathrm{MHz}, \mathrm{CDCl}_{3}\right) \delta$ : $7.39 \sim 7.36(\mathrm{~m}, 4 \mathrm{H}), 7.34 \sim 7.30(\mathrm{~m}, 1 \mathrm{H}), 6.64(\mathrm{~s}, 1 \mathrm{H})$, $4.20 \sim 4.14(\mathrm{~m}, 1 \mathrm{H}), 3.90(\mathrm{~s}, 3 \mathrm{H}), 2.65 \sim 2.60(\mathrm{~m}, 1 \mathrm{H})$, $2.51 \sim 2.46(\mathrm{~m}, 1 \mathrm{H}), 2.06 \sim 1.95(\mathrm{~m}, 2 \mathrm{H}), 1.77(\mathrm{dd}, J=6.6$, $0.5 \mathrm{~Hz}, 3 \mathrm{H}) ;{ }^{13} \mathrm{C}$ NMR $\left(151 \mathrm{MHz}, \mathrm{CDCl}_{3}\right) \delta: 164.0$ (t, $J=$ $34.8 \mathrm{~Hz}), 134.9,131.5$ (t, $J=5.9 \mathrm{~Hz}), 130.6$ (t, $J=23.5$ $\mathrm{Hz}), 130.1,129.3,127.7,113.6(\mathrm{t}, J=251.7 \mathrm{~Hz}), 53.7$, 50.5, 39.7, $31.4(\mathrm{t}, J=3.4 \mathrm{~Hz}), 26.5 ;{ }^{19} \mathrm{~F}$ NMR $(565 \mathrm{MHz}$, $\left.\mathrm{CDCl}_{3}\right) \delta$ : -99.2 ; HRMS (ESI) calcd for $\mathrm{C}_{15} \mathrm{H}_{17} \mathrm{BrF}_{2}-$ $\mathrm{O}_{2} \mathrm{SNa}(\mathrm{M}+\mathrm{Na})^{+}$400.9993, found 400.9989 .

2-三氟甲基-1-苯硫基-5-溴已烯(3ah): $28 \mathrm{mg}$, 黄色 液体, 产率 42\%. ${ }^{1} \mathrm{H}$ NMR $\left(600 \mathrm{MHz}, \mathrm{CDCl}_{3}\right) \delta: 7.45(\mathrm{~d}$, $J=7.4 \mathrm{~Hz}, 2 \mathrm{H}), 7.38(\mathrm{t}, J=7.4 \mathrm{~Hz}, 2 \mathrm{H}), 7.34$ (t, $J=7.3$ $\mathrm{Hz}, 1 \mathrm{H}), 6.68(\mathrm{~s}, 1 \mathrm{H}), 4.16 \sim 4.07(\mathrm{~m}, 1 \mathrm{H}), 2.61 \sim 2.48(\mathrm{~m}$, $1 \mathrm{H}), 2.47 \sim 2.29(\mathrm{~m}, 1 \mathrm{H}), 2.03 \sim 1.88(\mathrm{~m}, 2 \mathrm{H}), 1.74(\mathrm{~d}, J=$ $6.7 \mathrm{~Hz}, 3 \mathrm{H}) ;{ }^{13} \mathrm{C}$ NMR $\left(151 \mathrm{MHz}, \mathrm{CDCl}_{3}\right) \delta: 134.9$ (q, $J=$ $3.1 \mathrm{~Hz}), 130.9,129.5,128.1,127.15$ (q, $J=574.5 \mathrm{~Hz})$, 123.51 (q, $J=29.7 \mathrm{~Hz}), 50.1,39.5,31.1(\mathrm{q}, J=1.8 \mathrm{~Hz})$, $26.5 ;{ }^{19} \mathrm{~F}$ NMR (565 MHz, $\mathrm{CDCl}_{3}$ ) $\delta:-61.5$; MS (EI) $\mathrm{m} / \mathrm{z}$ 
(\%): $340\left(\mathrm{M}^{+},{ }^{81} \mathrm{Br}, 1\right), 338\left(\mathrm{M}^{+},{ }^{79} \mathrm{Br}, 24\right), 258$ (24), 217 (100), 189 (3), 184 (62).

2-三氟甲基-1-苯硫基-5-碘已烯(3ai)：23 mg, 黄色 液体, 产率 $30 \% .{ }^{1} \mathrm{H} \mathrm{NMR}\left(600 \mathrm{MHz}, \mathrm{CDCl}_{3}\right) \delta: 7.48(\mathrm{~d}$, $J=7.2 \mathrm{~Hz}, 2 \mathrm{H}), 7.40$ (t, $J=7.4 \mathrm{~Hz}, 2 \mathrm{H}), 7.37$ (d, $J=7.2$ $\mathrm{Hz}, 1 \mathrm{H}), 6.72(\mathrm{~s}, 1 \mathrm{H}), 4.35 \sim 4.01(\mathrm{~m}, 1 \mathrm{H}), 2.75 \sim 2.50(\mathrm{~m}$, 1H), $2.42 \sim 2.27(\mathrm{~m}, 1 \mathrm{H}), 1.98(\mathrm{~d}, J=6.8 \mathrm{~Hz}, 3 \mathrm{H}), 1.94$ $(\mathrm{dd}, J=9.6,4.8 \mathrm{~Hz}, 1 \mathrm{H}), 1.83 \sim 1.71(\mathrm{~m}, 1 \mathrm{H}) ;{ }^{13} \mathrm{C} \mathrm{NMR}$ $\left(151 \mathrm{MHz}, \mathrm{CDCl}_{3}\right) \delta: 134.90(\mathrm{q}, J=3.0 \mathrm{~Hz}), 131.0,129.4$, $128.1,123.57$ (q, $J=185.5 \mathrm{~Hz}), 123.35$ (q, $J=30.0 \mathrm{~Hz})$, 41.1, 33.1, 28.9, 28.5; ${ }^{19} \mathrm{~F}$ NMR (565 MHz, $\left.\mathrm{CDCl}_{3}\right) \delta$ : -61.5; MS (EI) $m / z(\%): 386\left(\mathrm{M}^{+}, 18\right), 259$ (29), 217 (100), 189 (1), 184 (49).

1-苯硫基-2-对甲苯磺酰基-5-氯己烯(3aj)：43 mg, 黄色液体, 产率 57\%. ${ }^{1} \mathrm{H}$ NMR (600 $\left.\mathrm{MHz}, \mathrm{CDCl}_{3}\right) \delta: 7.92$ $(\mathrm{d}, J=8.1 \mathrm{~Hz}, 2 \mathrm{H}), 7.45$ (d, $J=7.1 \mathrm{~Hz}, 2 \mathrm{H}), 7.36$ (dd, $J=$ 9.8, $5.9 \mathrm{~Hz}, 5 \mathrm{H}), 7.02(\mathrm{~s}, 1 \mathrm{H}), 3.93 \sim 3.99(\mathrm{~m}, 1 \mathrm{H}), 2.58 \sim$ $2.50(\mathrm{~m}, 1 \mathrm{H}), 2.45(\mathrm{~s}, 3 \mathrm{H}), 2.44 \sim 2.37(\mathrm{~m}, 1 \mathrm{H}), 2.02 \sim$ $2.08(\mathrm{~m}, 1 \mathrm{H}), 1.76 \sim 1.82(\mathrm{~m}, 1 \mathrm{H}), 1.47(\mathrm{~d}, J=6.5 \mathrm{~Hz}$, $3 \mathrm{H}) ;{ }^{13} \mathrm{C} \mathrm{NMR}\left(151 \mathrm{MHz}, \mathrm{CDCl}_{3}\right) \delta: 144.8,142.6,137.6$, $135.7,132.6,131.1,129.9,129.5,128.5,127.5,57.5,39.4$, $30.5,25.4,21.7$; HRMS (ESI) calcd for $\mathrm{C}_{19} \mathrm{H}_{21} \mathrm{ClO}_{2} \mathrm{~S}_{2} \mathrm{Na}$ $(\mathrm{M}+\mathrm{Na})^{+}$403.0564, found 403.0568.

辅助材料(Supporting Information) 化合物 3 的 ${ }^{1} \mathrm{H}$ $\mathrm{NMR} 、{ }^{13} \mathrm{C} N \mathrm{NMR}$ 和 ${ }^{19} \mathrm{~F}$ NMR 谱图. 这些材料可以免费 从本刊网站(http://sioc-journal.cn/)上下载

\section{References}

[1] For selected reviews, see: (a) Purser, S.; Moore, P. R. Swallow, S.; Gouverneur, V. Chem. Soc. Rev. 2008, 37, 320. (b) O'Hagan, D. Chem. Soc. Rev. 2008, 37, 308.

[2] (a) Burke, T. R.; Lee, K. Acc. Chem. Res. 2003, 36, 426.

(b) Meanwell, N. A. J. Med. Chem. 2011, 54, 2529.

(c) An, L.; Tong, F.; Zhang, X. Acta Chim. Sinica 2018, 76, 977 (in Chinese).

(安伦, 童非非, 张新刚, 化学学报, 2018, 76, 977.)

(d) Tao, X.; Sheng, R.; Bao, K.; Wang, Y.; Jin, Y. Chin. J. Org. Chem. 2019, 39, 2726 (in Chinese).

(陶雪芬，盛荣，鲍勏，王玉新，金银秀，有机化学，2019，39， 2726.)

(e) Dai, J.; Lei, W.; Liu, Q. Acta Chim. Sinica 2019, 77, 911 (in Chinese).

(戴建玲, 雷文龙, 刘强, 化学学报, 2019, 77, 911.)

[3] (a) Long, Z.-Y.; Chen, Q.-Y. J. Org. Chem. 1999, 64, 4775.

(b) Huang, X.-T.; Chen, Q.-Y. J. Org. Chem. 2001, 66, 4651.

(c) Ke, M.; Feng, Q.; Yang, K.; Song, Q. Org. Chem. Front. 2016, 3,150 .

(d) Feng, X.; Wang, X.; Chen, H.; Tang, X.; Guo, M.; Zhao, W.; Wang, G. Org. Biomol. Chem. 2018, 16, 2841.

(e) Li, K.-K.; Zhang, X.-X.; Chen, J.-C.; Gang, Y.; Yang, C.-H.;
Zhang, K.-Y.; Zhou, Y.-Y.; Fan, B.-M. Org. Lett. 2019, 21, 9914.

[4] (a) He, Y.-T.; Wang, Q.; Li, L.-H.; Liu, X.-Y.; Xu, P.-F.; Liang, Y.-M. Org. Lett. 2015, 17, 5188.

(b) He, Y.-T.; Li, L.-H.; Wang, Q.; Wu, W.; Liang, Y.-M. Org. Lett. 2016, 18, 5158.

(c) Wang, Q.; Zheng, L.; He, Y.-T.; Liang, Y.-M. Chem. Commun. 2017, 53, 2814.

(d) Zhang, Y.; Zhang, J.; Hu, B.; Ji, M.; Ye, S.; Zhu, G. Org. Lett. 2018, 20, 2988.

(e) Liang, J.-Q.; Huang, G.-Z; Peng, P.; Zhang, T.-Y.; Wu, J.-J.; Wu, F.-H. Adv. Synth. Catal. 2018, 360, 2221.

[5] Zhang, B.-S.; Gao, L.-Y.; Zhang, Z.; Wen, Y.-H.; Liang, Y.-M. Chem. Commun. 2018, 54, 1185.

[6] Xiang, Y.; Li, Y.; Kuang, Y.; Wu, J. Chem.-Eur. J. 2017, 23, 1032.

[7] (a) Wang, S.; Zhang, J.; Kong, L.; Tan, Z.; Bai, Y.; Zhu, G. Org. Lett. 2018, 20, 5631.

(b) Guo, W.-H.; Zhao, H.-Y.; Luo, Z.-J.; Zhang, S.; Zhang, X. ACS Catal. 2019, 9, 38 .

[8] Li, Y.; Li, H.; Hu, J. Tetrahedron 2009, 65, 478.

[9] Xu, T.; Cheung, C. W.; Hu, X. Angew. Chem., Int. Ed. 2014, 53, 4910.

[10] Zhong, J.-J.; Yang, C.; Chang, X.-Y.; Zou, C.; Lu, W.; Che, C.-M. Chem. Commun. 2017, 53, 8948

[11] Li, G.; Cao, Y.-X.; Luo, C.-G.; Su, Y.-M.; Li, Y.; Lan, Q.; Wang, X.-S. Org. Lett. 2016, 18, 4806.

[12] Wu, G.; von Wangelin, A. J. V. Chem. Sci. 2018, 9, 1795.

[13] Shang, T.; Zhang, J.; Zhang, Y.; Zhang, F.; Li, X.-S.; Zhu, G. Org. Lett. 2020, 22, 3667.

[14] Xiong, Z.; Zhang, F.; Yu, Y.; Tan, Z.; Zhu, G. Org. Lett. 2020, 22, 4088.

[15] For selected reviews on photocatalysis, see: (a) Narayanam, J. M. R.; Stephenson, C. R. J. Chem. Soc. Rev. 2011, 40, 102.

(b) Xuan, J.; Xiao, W.-J. Angew. Chem., Int. Ed. 2012, 51, 6828.

(c) Prier, C. K.; Rankic, D. A.; MacMillan, D. W. C. Chem. Rev. 2013, 113, 5322 .

(d) Matsui, J. K.; Lang, S. B.; Heitz, D. R.; Molander, G. A. ACS Catal. 2017, 7, 2563.

[16] For selected reports on remote C-H halogention, see: (a) Kundu, R.; Ball, Z. T. Org. Lett. 2010, 12, 2460.

(b) Liu, T.; Myers, M. C.; Yu, J.-Q. Angew. Chem., Int. Ed. 2017, 56,306

(c) Herron, A. N.; Liu, D.; Xia, G.; Yu, J.-Q. J. Am. Chem. Soc. 2020, 142, 2766.

(d) Short, M. A.; Blackburn, J. M.; Roizen, J. L. Angew. Chem., Int. Ed. 2018, 57, 296.

[17] (a) Yang, Z.; Chen, X.; Kong, W.; Xia, S.; Zheng, R.; Luo, F.; Zhu, G. Org. Biomol. Chem. 2013, 11, 2175.

(b) Zhu, G.; Kong, W.; Feng, H.; Qian, Z. J. Org. Chem. 2014, 79, 1786.

[18] Nie, X.; Cheng, C.; Zhu, G. Angew. Chem., Int. Ed. 2017, 56, 1898.

[19] (a) Jin, W.; Wu, M.; Xiong, Z.; Zhu, G. Chem. Commun. 2018, 54, 7924.

(b) Wan, Y.; Shang, T.; Lu, Z.; Zhu, G. Org. Lett. 2019, 21, 4187.

[20] For selected reports on HAT of vinyl radicals, see: (a) Curran, D. P.; Shen, W. J. Am. Chem. Soc. 1993, 115, 6051.

(b) Bogen, S.; Malacria, M. J. Am. Chem. Soc. 1996, 118, 3992.

(c) Hu, M.; Fan, J.-H.; Liu, Y.; Ouyang, X.-H.; Song, R.-J.; Li, J.-H. Angew. Chem., Int. Ed. 2015, 54, 9577.

(d) Qiu, J.-K.; Jiang, B.; Zhu, Y.-L.; Hao, W.-J.; Wang, D.-C.; Sun, J.; Wei, P.; Tu, S.-J.; Li, G. J. Am. Chem. Soc. 2015, 137, 8928.

(e) Huang, L.; Ye, L.; Li, X.-H.; Li, Z.-L.; Lin, J.-S.; Liu, X.-Y. Org. Lett. 2016, 18, 5284. 
(f) Gloor, C. S.; Dénès, F.; Renaud, P. Angew. Chem., Int. Ed. 2017 , 56, 13329.

(g) An, X.-D.; Jiao, Y.-Y.; Zhang, H.; Gao, Y.; Yu, S. Org. Lett. 2018, 20, 401.

(h) Ratushnyy, M.; Parasram, M.; Wang, Y.; Gevorgyan, V. Angew. Chem., Int. Ed. 2018, 57, 2712.

(i) Wu, S.; Wu, X.; Wang, D.; Zhu, C. Angew. Chem., Int. Ed. 2019, $58,1499$. (j) Yang, S.; Wu, X.; Wu, S.; Zhu, C. Org. Lett. 2019, 21, 4837.

(k) Liu, T.; Qu, C.; Xie, J.; Zhu, C. Chin. J. Org. Chem. 2019, 39, 1613 (in Chinese).

(刘涛, 屈川华, 谢劲, 朱成建, 有机化学, 2019, 39, 1613.)

(1) Li, H.; Guo, L.; Feng, X.; Huo, L.; Zhu, S.; Chu, L. Chem. Sci. 2020, $11,4904$.

(m) Wu, S.; Wu, X.; Wu, Z.; Zhu, C. Sci. China: Chem. 2019, 62, 1507.

(Cheng, F.) 